\title{
The configurational measure on mutually avoiding SLE paths
}

\author{
Michael J. Kozdron* \\ Department of Mathematics \& Statistics, College West 307.14 \\ University of Regina, Regina, SK S4S 0A2 Canada \\ kozdron@math.uregina.ca \\ Gregory F. Lawler ${ }^{\dagger}$ \\ Department of Mathematics, 310 Mallot Hall \\ Cornell University, Ithaca, NY 14853 USA \\ lawler@math.cornell.edu
}

\begin{abstract}
We define multiple chordal SLEs in a simply connected domain by considering a natural configurational measure on paths. We show how to construct these measures so that they are conformally covariant and satisfy certain boundary perturbation and Markov properties, as well as a cascade relation. As an example of our construction, we derive the scaling limit of Fomin's identity in the case of two paths directly; that is, we prove that the probability that an $\mathrm{SLE}_{2}$ and a Brownian excursion do not intersect can be given in terms of the determinant of the excursion hitting matrix. Finally, we define the $\lambda$-SAW, a one-parameter family of measures on self-avoiding walks on $\mathbb{Z}^{2}$.
\end{abstract}

2000 Mathematics Subject Classification. 60G50, 60J45, 60J65

Key words and phrases. Schramm-Loewner evolution, loop-erased random walk, self-avoiding random walk, loop measures, Fomin's identity, conformal Markov property, boundary scaling exponent.

\footnotetext{
*Research supported in part by the Natural Sciences and Engineering Research Council of Canada.

$\dagger$ Research supported by the National Science Foundation grant DMS-0405021.
} 


\section{Introduction}

The Schramm-Loewner evolution (SLE) is a powerful tool for describing continuum limits that arise in critical two-dimensional models in statistical physics. The starting point [16] is to write down some properties that the continuum limit is expected to satisfy and then to show that this leaves only a one-parameter family of possibilities. This works beautifully in the case of one path in a simply connected domain.

For multiple paths or non-simply connected domains, the approach in [16] is not sufficient to determine what the natural definition(s) should be. Essentially the conformal Markov property, which determines the distribution of a single SLE path in simply connected domains, is not sufficient to describe the more complicated situations. A number of authors have tried to consider these processes using infinitesimal descriptions. In this paper, we would like to try to suggest that this is not the most natural approach, and that instead one should look at configurational measures on paths. What we do here is describe the configurational measure associated to multiple chordal SLEs in a simply connected domain. This construction is based on the work in [10] and is also discussed in [3]. For a statistical mechanics argument describing multiple SLEs, consult [1]. Nonetheless, it still seems useful to describe the configurational measure in some detail.

We will start by writing down a number of properties that we expect the measure to have. We will not worry at the moment whether some of these properties are redundant or consistent. Let $D$ be a Jordan domain, and let $z_{1}, \ldots, z_{n}, w_{n}, \ldots, w_{1}$ be $2 n$ distinct points on $\partial D$ in counterclockwise order. We write $\mathbf{z}=\left(z_{1}, \ldots, z_{n}\right), \mathbf{w}=\left(w_{1}, \ldots, w_{n}\right)$, and say that $D$ is (locally) analytic at $\mathbf{z}, \mathbf{w}$ if conformal maps sending $D$ to the unit disk $\mathbb{D}$ can be extended analytically to neighborhoods of $z_{1}, \ldots, z_{n}, w_{n}, \ldots, w_{1}$. We fix $b \in \mathbb{R}$, a parameter that is called the boundary scaling exponent or boundary conformal weight. We want to define a measure $Q_{D, b, n}(\mathbf{z}, \mathbf{w})$ on mutually avoiding $n$-tuples of simple paths $\left(\gamma^{1}, \ldots, \gamma^{n}\right)$ in $D$. More precisely, $\gamma^{j}$ is an equivalence class of curves such that there is a representative $\gamma^{j}:[0,1] \rightarrow \mathbb{C}$ which is simple, $\gamma^{j}(0)=z_{j}, \gamma^{j}(1)=w_{j}$, and $\gamma^{j}(0,1) \subset D$. We will write just $\gamma^{j}$ for $\gamma^{j}(0,1)$. When we refer to measures on curves in this paper, we will implicitly mean measures on equivalence classes of curves under equivalence by reparametrization; consult, for example, [5] or [8].

- Conformal Covariance. If $D$ is analytic at $\mathbf{z}, \mathbf{w}$, then $Q_{D, b, n}(\mathbf{z}, \mathbf{w})$ is a non-zero, finite measure supported on $n$-tuples $\left(\gamma^{1}, \ldots, \gamma^{n}\right)$ where $\gamma^{j}$ is a simple curve in $D$ connecting $z_{j}$ and $w_{j}$ and

$$
\gamma^{j} \cap \gamma^{k}=\emptyset, \quad 1 \leq j<k \leq n
$$

Moreover, if $f: D \rightarrow f(D)$ is a conformal transformation and $f(D)$ is analytic at $f(\mathbf{z})$, $f(\mathbf{w})$, then

$$
f \circ Q_{D, b, n}(\mathbf{z}, \mathbf{w})=\left|f^{\prime}(\mathbf{z})\right|^{b}\left|f^{\prime}(\mathbf{w})\right|^{b} Q_{f(D), b, n}(f(\mathbf{z}), f(\mathbf{w}))
$$

where $f(\mathbf{z})=\left(f\left(z_{1}\right), \ldots, f\left(z_{n}\right)\right)$ and $f^{\prime}(\mathbf{z})=f^{\prime}\left(z_{1}\right) \cdots f^{\prime}\left(z_{n}\right)$; see Figure 1

If we write

$$
Q_{D, b, n}(\mathbf{z}, \mathbf{w})=H_{D, b, n}(\mathbf{z}, \mathbf{w}) \mu_{D, b, n}^{\#}(\mathbf{z}, \mathbf{w}),
$$


where $H_{D, b, n}(\mathbf{z}, \mathbf{w})=\left|Q_{D, b, n}(\mathbf{z}, \mathbf{w})\right|$ and $\mu_{D, b, n}^{\#}(\mathbf{z}, \mathbf{w})$ is a probability measure, then the conformal covariance condition (II) becomes the scaling rule for $H$,

$$
H_{D, b, n}(\mathbf{z}, \mathbf{w})=\left|f^{\prime}(\mathbf{z})\right|^{b}\left|f^{\prime}(\mathbf{w})\right|^{b} H_{f(D), b, n}(f(\mathbf{z}), f(\mathbf{w})),
$$

and the conformal invariance rule for $\mu^{\#}$, namely

$$
f \circ \mu_{D, b, n}^{\#}(\mathbf{z}, \mathbf{w})=\mu_{f(D), b, n}^{\#}(f(\mathbf{z}), f(\mathbf{w})) .
$$

Since $\mu^{\#}$ is a conformal invariant, we can define $\mu_{D, b, n}^{\#}(\mathbf{z}, \mathbf{w})$ even if the boundaries are not smooth at $\mathbf{z}, \mathbf{w}$. (Compare this with the construction of the Brownian excursion measure in [5].) We will write $Q_{D, b}(z, w)=Q_{D, b, 1}(z, w)$ and similarly for $H_{D, b}(z, w)$ and $\mu_{D, b}^{\#}(z, w)$.
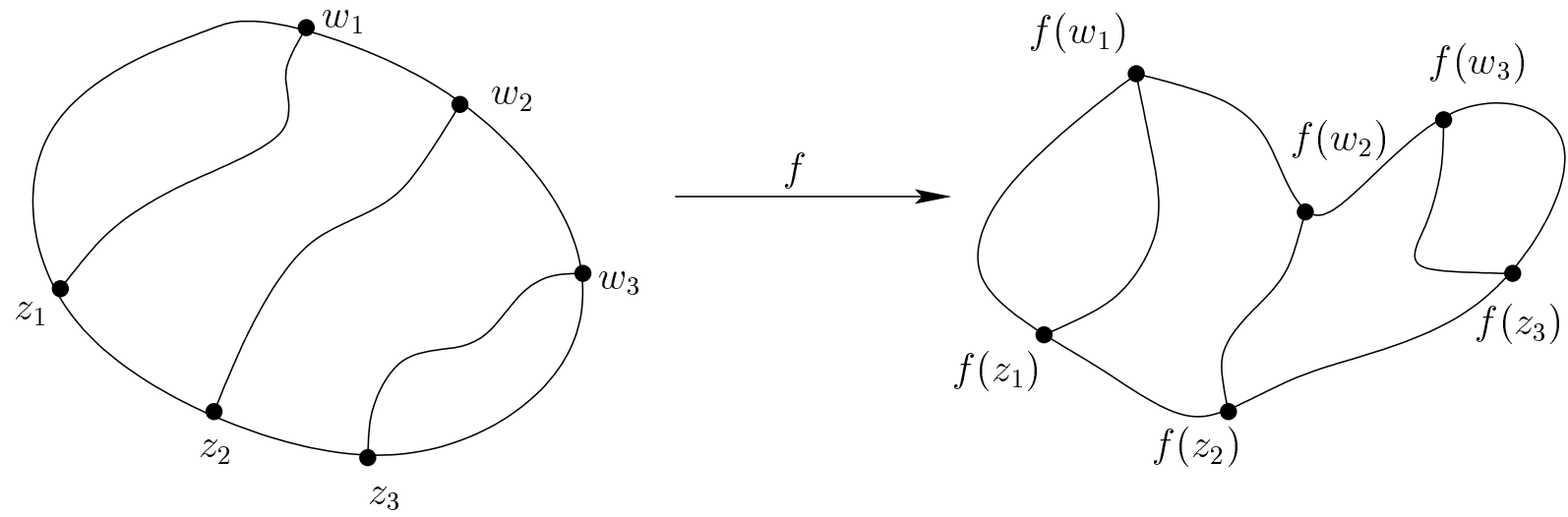

Figure 1: Conformal Covariance

The next condition describes what happens when we remove part of the domain.

- Boundary Perturbation. Suppose $D \subset D^{\prime}$ are Jordan domains and $\partial D, \partial D^{\prime}$ agree and are analytic in neighborhoods of $\mathbf{z}, \mathbf{w}$. Then $Q_{D, b, n}(\mathbf{z}, \mathbf{w})$ is absolutely continuous with respect to $Q_{D^{\prime}, b, n}(\mathbf{z}, \mathbf{w})$. Moreover, the Radon-Nikodym derivative

$$
Y_{D, D^{\prime}, b, n}(\mathbf{z}, \mathbf{w})=\frac{d Q_{D, b, n}(\mathbf{z}, \mathbf{w})}{d Q_{D^{\prime}, b, n}(\mathbf{z}, \mathbf{w})}
$$

is a conformal invariant. In other words, if $f: D^{\prime} \rightarrow f\left(D^{\prime}\right)$ is a conformal map that extends analytically in neighborhoods of $\mathbf{z}, \mathbf{w}$, then

$$
Y_{f(D), f\left(D^{\prime}\right), b, n}(f(\mathbf{z}), f(\mathbf{w}))(f \circ \bar{\gamma})=Y_{D, D^{\prime}, b, n}(\mathbf{z}, \mathbf{w})(\bar{\gamma})
$$

where $\bar{\gamma}=\left(\gamma^{1}, \ldots, \gamma^{n}\right)$ and $f \circ \bar{\gamma}=\left(f \circ \gamma^{1}, \ldots, f \circ \gamma^{n}\right)$; see Figure 2

As with $\mu_{D, b, n}^{\#}(\mathbf{z}, \mathbf{w})$, the last condition (4) implies that $Y_{D, D^{\prime}, b, n}(\mathbf{z}, \mathbf{w})$ is well-defined even if the boundaries are not smooth at $\mathbf{z}, \mathbf{w}$.

The third relation relates the measures for different $n$. 


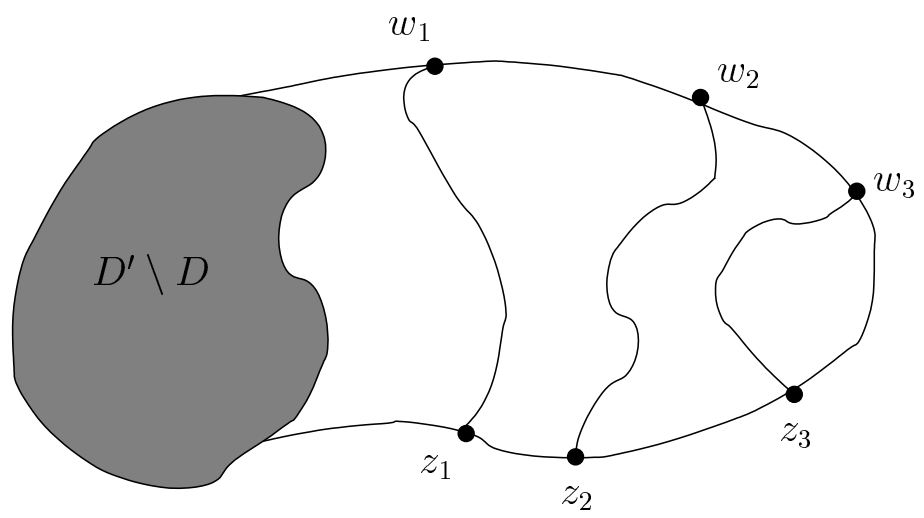

Figure 2: Boundary Perturbation

- Cascade Relation. Let

$$
\begin{gathered}
\hat{\mathbf{z}}=\left(z_{1}, \ldots, z_{j-1}, z_{j+1}, \ldots, z_{n}\right), \quad \hat{\mathbf{w}}=\left(w_{1}, \ldots, w_{j-1}, w_{j+1}, \ldots, w_{n}\right), \\
\hat{\gamma}=\left(\gamma^{1}, \ldots, \gamma^{j-1}, \gamma^{j+1}, \ldots, \gamma^{n}\right) .
\end{gathered}
$$

The marginal distribution on $\hat{\gamma}$ induced by $Q_{D, b, n}(\mathbf{z}, \mathbf{w})$ is absolutely continuous with respect to $Q_{D, b, n-1}(\hat{\mathbf{z}}, \hat{\mathbf{w}})$ with Radon-Nikodym derivative $H_{\hat{D}, b}\left(z_{j}, w_{j}\right)$. Here $\hat{D}$ is the subdomain of $D \backslash \hat{\gamma}$ whose boundary includes $z_{j}, w_{j}$; see Figure 3 .

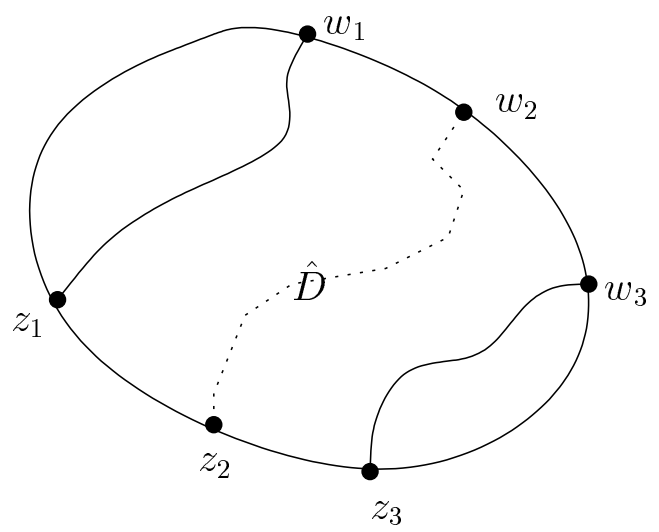

Figure 3: Cascade Relation. $\hat{z}=\left(z_{1}, z_{3}\right), \hat{w}=\left(w_{1}, w_{3}\right)$.

In order to construct such a collection of measures it is useful to include a fourth condition. This condition is stated in terms of $\mu_{D, b}^{\#}$ rather than $Q_{D, b}$.

- Markov Property. In the measure $\mu_{D, b}^{\#}(z, w)$, the conditional distribution on $\gamma$ given an initial segment $\gamma[0, t]$ is $\mu_{D \backslash \gamma[0, t], b}^{\#}(\gamma(t), w)$; see Figure 4 . 


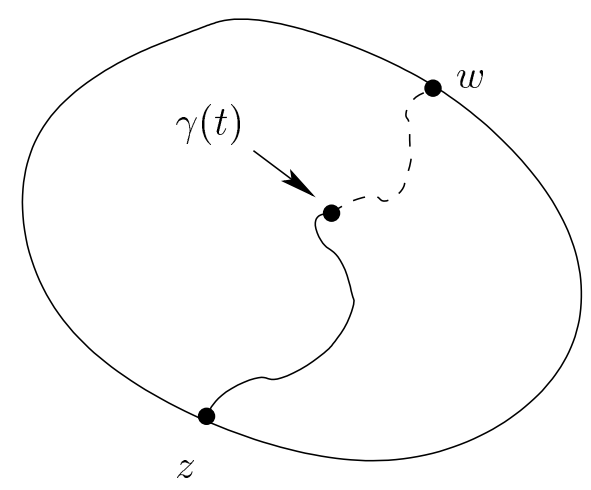

Figure 4: Markov Property

We have stated this condition in a way that does not use two dimensions and conformal invariance. The conformal Markov property is the combination of the Markov property and (3). Schramm [16] showed that there is a one-parameter family of measures, which he parametrized by $\kappa$, satisfying the conformal Markov property. While these measures are well-defined for $\kappa>0$, they are supported on simple curves only for $0<\kappa \leq 4$; see [8, Proposition 6.9]. Therefore, we will restrict our consideration only to $\kappa \leq 4$. The scaling rule (2) implies that, up to an arbitrary multiplicative constant,

$$
H_{b}(x, y)=H_{\mathbb{H}, b}(x, y)=H_{\mathbb{H}, b, 1}(x, y)=|y-x|^{-2 b} .
$$

We will continue to write $H_{b}$ for $H_{\mathbb{H}, b}\left(=H_{\mathbb{H}, b, 1}\right)$ where $\mathbb{H}=\{z \in \mathbb{C}: \Im(z)>0\}$ is the upper half plane. We will choose the constant to be 1 so that (5) holds. Given this, the measures $Q_{D, b, n}(\mathbf{z}, \mathbf{w})$ can be determined for all simply connected $D$ and $\mathbf{z}, \mathbf{w}$ at which $\partial D$ is analytic by a combination of conformal mapping and the cascade relation. The question is whether or not we can find $b, \kappa$ such that all four of the properties above hold. The answer is yes, assuming we choose $\kappa=\kappa(b)$ satisfying

$$
\kappa=\frac{6}{2 b+1} \quad \text { so that } \quad b=\frac{6-\kappa}{2 \kappa} .
$$

Notice that $b$ decreases from $\infty$ to $1 / 4$ as $\kappa$ increases from 0 to 4 which puts the restriction $b \geq 1 / 4$ on the scaling exponent. There are four other parameters that can be used to parametrize this family, namely

$$
a=\frac{2}{\kappa}=\frac{2 b+1}{3}, \quad \lambda=\frac{(3 a-1)(4 a-3)}{2 a}, \quad c=-2 \lambda, \quad \text { and } \quad d=1+\frac{1}{4 a} .
$$

These parameters have interpretations which can be summarized as follows.

- $b \in[1 / 4, \infty)$ is the boundary scaling exponent or boundary conformal weight.

- $\kappa \in(0,4]$ is the variance of the driving Brownian motion in $\mathrm{SLE}_{\kappa}$ if the half-plane capacity at time $t$ is $2 t$. 
- $a \in[1 / 2, \infty)$ and at is the half-plane capacity at time $t$ for $\mathrm{SLE}_{\kappa}$ if the driving Brownian motion is chosen to have variance 1 .

- $\lambda \in[-1 / 2, \infty)$ is the exponent of the Brownian loop measure that arises in the RadonNikodym derivatives.

- $c \in(-\infty, 1]$ is the central charge. We will not use this parameter in this paper, but $c$ comes up naturally in representations of conformal field theories.

- $d \in(1,3 / 2]$ is the Hausdorff dimension of the paths. This is also a parameter that will not be discussed in this paper.

We will tend to use $b$ as our parameter, but any of the quantities above could equally well be used.

We have defined the measure $Q_{D, b, n}(\mathbf{z}, \mathbf{w})$ for fixed $\mathbf{z}, \mathbf{w}$. However, if $D$ has a piecewise analytic boundary, we can define

$$
Q_{D, b, n}=\int_{(\partial D)^{n} \times(\partial D)^{n}} Q_{D, b, n}(\mathbf{z}, \mathbf{w}) d \mathbf{z} d \mathbf{w} .
$$

In the case $b=1$, the scaling rule implies that $Q_{D, 1, n}$ is conformally invariant.

\section{Preliminaries}

\section{$2.1 \mathrm{SLE}_{\kappa}$}

We review the definition of chordal $\mathrm{SLE}_{\kappa}$ in simply connected domains. We will only consider the case $\kappa \leq 4$ in this paper, and let $a=2 / \kappa$ as in (6). Then $\mathrm{SLE}_{\kappa}$ in $\mathbb{H}$ from 0 to $\infty$ is the random curve $\gamma:(0, \infty) \rightarrow \mathbb{H}$ with $\gamma(0+)=0$ satisfying the following. Let $g_{t}: \mathbb{H} \backslash \gamma(0, t] \rightarrow \mathbb{H}$ be the unique conformal transformation with $g_{t}(z)-z=o(1)$ as $z \rightarrow \infty$. Then $g_{t}$ satisfies the chordal Loewner differential equation

$$
\dot{g}_{t}(z)=\frac{a}{g_{t}(z)-U_{t}}, \quad g_{0}(z)=z
$$

where the driving function $U_{t}$ is a standard one-dimensional Brownian motion. The curve has been parametrized so that $\gamma(0, t]$ has half-plane capacity at, i.e., so that $g_{t}$ has the expansion

$$
g_{t}(z)=z+\frac{a t}{z}+O\left(|z|^{-2}\right), \quad z \rightarrow \infty .
$$

If $D$ is a simply connected domain and $z, w$ are distinct boundary points of $D$, then chordal $\mathrm{SLE}_{\kappa}$ from $z$ to $w$ is defined to be the conformal image of $\mathrm{SLE}_{\kappa}$ in $\mathbb{H}$ under a conformal transformation taking 0 to $z$ and $\infty$ to $w$. We consider this as a probability measure on curves modulo reparametrization, and denote this probability measure by $\mu_{D, b}^{\#}(z, w)$.

There are a number of special values for $\kappa$. In this paper we will look at special properties of $\kappa=2$. As we now show, the stochastic process $\dot{g}_{t}(z)$ is a martingale iff $\kappa=2$ (equivalently, $a=1)$. 
Proposition 2.1. Suppose that $g_{t}(z)$ satisfies the chordal Loewner equation (7). If $M_{t}=$ $M_{t}^{z, a}=\dot{g}_{t}(z)$, then $M_{t}$ is a martingale if and only if $a=1$.

Proof. If $Z_{t}=g_{t}(z)-U_{t}$, then a simple calculation using Itô's formula shows that

$$
d Z_{t}=\frac{a}{Z_{t}} d t-d U_{t}
$$

so that $Z_{t}$ is a Bessel process with $d\langle Z\rangle_{t}=d t$. Using Itô's formula again therefore gives

$$
d M_{t}=d\left(\frac{a}{Z_{t}}\right)=-\frac{a}{Z_{t}^{2}} d Z_{t}+\frac{a}{Z_{t}^{3}} d\langle Z\rangle_{t}=\frac{a}{Z_{t}^{2}} d U_{t}+\frac{a(1-a)}{Z_{t}^{3}} d t .
$$

Hence, $M_{t}$ is a martingale iff $a=1$, in which case $d M_{t}=M_{t}^{2} d U_{t}$.

\subsection{Brownian loop measure}

We review the Brownian loop measure as introduced in [14]; see also [8, Section 5.7]. (That paper considered the loop soup, which is a Poissonian realization of the loop measure. This loop soup is very useful heuristically but is not needed for the definition of the measure.) A rooted loop of time duration $t$ is a continuous function $\eta:[0, t] \rightarrow \mathbb{C}$ with $\eta(0)=\eta(t)$. It can also be considered as an ordered pair $(t, \eta)$ where $\eta:(-\infty, \infty) \rightarrow \mathbb{C}$ is a continuous function with period $t$. An unrooted loop is an equivalence class of rooted loops under the equivalence $(t, \eta) \sim\left(t, \eta_{s}\right)$ for all $s$ where $\eta_{s}(r)=\eta(s+r)$. Each rooted loop can be written uniquely as $(t, z, \bar{\eta})$ where $t>0$ is the time duration, $z$ is the root, and $\bar{\eta}$ is a loop of time duration 1 rooted at the origin. More specifically, $\eta$ is given by translation and Brownian scaling, $\eta(s)=z+t^{1 / 2} \bar{\eta}(s / t)$. The rooted Brownian loop measure is given by choosing $(t, z, \bar{\eta})$ from the measure

$$
\frac{1}{2 \pi t} d t \times \text { area } \times \nu_{B B}
$$

where $\nu_{B B}$ denotes the probability measure associated to Brownian bridges. The Brownian loop measure is the measure on unrooted loops obtained from this measure by "forgetting the loops." The loop measure in a domain $D$ is exactly this measure restricted to loops that stay in the domain. An important fact is that the Brownian loop measure is conformally invariant: if $f: D \rightarrow D^{\prime}$ is a conformal transformation, then the image of the loop measure on $D$ by $f$ is the loop measure on $D^{\prime}$. (If we are worrying about the parametrization of the loops, we have to change the parametrization by the usual rule for conformal invariance of Brownian motion. In this paper, the parametrization will not be important, only the traces of the loops.) The Brownian loop measure can also be obtained as the scaling limit of a simple measure on random walk loops; see Section 5.1

If $D \subsetneq \mathbb{C}$ is simply connected and $V_{1}, V_{2}$ are closed sets, we let $m\left(D ; V_{1}, V_{2}\right)<\infty$ denote the Brownian loop measure of loops in $D$ that intersect both $V_{1}$ and $V_{2}$. If $f: D \rightarrow D^{\prime}$ is a conformal transformation, then $m\left(f(D) ; f\left(V_{1}\right), f\left(V_{2}\right)\right)=m\left(D ; V_{1}, V_{2}\right)$. The two main properties of the Brownian loop measure that we will use are conformal invariance, and the fact that if $D$ is simply connected, then $0<m\left(D ; V_{1}, V_{2}\right)<\infty$, provided that $V_{1}, V_{2}$ are disjoint closed sets with at least one $V_{i} \operatorname{compact}$; $\operatorname{dist}\left(V_{1}, V_{2}\right)>0$; and non-polar, i.e., such that with positive probability a Brownian motion starting at $z \in D$ hits both of these sets. 


\subsection{Boundary perturbation of SLE}

Suppose $D \subset \mathbb{H}$ is a simply connected domain such that $\mathbb{H} \backslash D$ is bounded, and $x \in \mathbb{R}$ with $\operatorname{dist}(x, \mathbb{H} \backslash D)>0$. For any such domain there is a unique conformal transformation $\Phi=\Phi_{D, x}: D \rightarrow \mathbb{H}$ such that $\Phi(x)=0, \Phi(\infty)=\infty, \Phi^{\prime}(\infty)=1$. Moreover, Schwarz reflection tells us how to extend $\Phi$ analytically in a neighborhood of $x$. Schramm [16] defined $\mathrm{SLE}_{\kappa}$ (modulo time parametrization) in $D$ from $x$ to $\infty$ to be the image of $\mathrm{SLE}_{\kappa}$ in $\mathbb{H}$ from 0 to $\infty$ under the conformal map $\Phi^{-1}$. By using properties of conformal mappings and Itô's formula (see, e.g., [8, Section 4.6]) one can give an equivalent formulation (see, e.g., [9, Section 4.3]). Let $\gamma$ and $g_{t}$ be as above, and let $D_{t}=g_{t}(D), \Phi_{t}=\Phi_{D_{t}, U_{t}}$. Consider the solution to (77) where the driving function $U_{t}$ satisfies the SDE

$$
d U_{t}=b \frac{\Phi_{t}^{\prime \prime}\left(U_{t}\right)}{\Phi_{t}^{\prime}\left(U_{t}\right)} d t+d B_{t}
$$

where $B_{t}$ is a standard one-dimensional Brownian motion. Then the distribution of the path $\gamma$ is exactly that of $\mathrm{SLE}_{\kappa}$ in $D$. In this formulation we have parametrized the paths so that $g_{t}$ satisfies (8). This is not the same parametrization as one would get if one started with an $\operatorname{SLE}_{\kappa}$ in $\mathbb{H}$ from 0 to $\infty$, say $\tilde{\gamma}$, and let $\gamma(t)=\Phi^{-1} \tilde{\gamma}(t)$. However, we only define $\operatorname{SLE}_{\kappa}$ in domains up to time reparametrization, so this is not an issue. In fact, we could have used (9) as the definition of $\mathrm{SLE}_{\kappa}$ in $D$, and then conformal invariance of the process would be a result.

There is a another equivalent way of defining $\mathrm{SLE}_{\kappa}$ in $D$ (for $\kappa \leq 4$ ) that comes from a martingale introduced in [10]. Let $\gamma$ be an $\mathrm{SLE}_{\kappa}$ in $\mathbb{H}$ from $x$ to $\infty$ parametrized as in (8). Let $T=T_{D}=\inf \{t>0: \gamma(t) \in \mathbb{H} \backslash D\}$. If $t<T$, then we can let $D_{t}$ and $\Phi_{t}$ be as in the previous paragraph. Define $Y_{t}$ by

$$
Y_{t}=1\{\gamma[0, t] \cap(\mathbb{H} \backslash D)=\emptyset\} \exp \left\{\frac{a \lambda}{12} \int_{0}^{t} S \Phi_{s}\left(U_{s}\right) d s\right\},
$$

where, as before,

$$
\lambda=\lambda(\kappa)=\frac{(3 a-1)(4 a-3)}{2 a}=\frac{(6-\kappa)(8-3 \kappa)}{4 \kappa},
$$

and $S$ denotes the Schwarzian derivative. In fact (see [10] and [14]),

$$
-\frac{a}{12} \int_{0}^{t} S \Phi_{s}\left(U_{s}\right) d s=m(\mathbb{H} ; \mathbb{H} \backslash D, \gamma(0, t]) .
$$

(The factor $a$ comes from the fact that $\gamma$ is parametrized as in (8).) Hence, we can write

$$
Y_{t}=1\{\gamma[0, t] \cap(\mathbb{H} \backslash D)=\emptyset\} \exp \{-\lambda m(\mathbb{H} ; \mathbb{H} \backslash D, \gamma(0, t])\}
$$

In [10] it was shown that $M_{t}=\Phi_{t}^{\prime}\left(U_{t}\right)^{b} Y_{t}$ is a local martingale. It is easy to check that $\Phi_{t}^{\prime}\left(U_{t}\right) \leq 1$ which implies that $M_{t}$ is a bounded martingale for $\lambda \geq 0(\kappa \leq 8 / 3)$. In this case, Girsanov's theorem can be used to find the effective SDE for paths weighted by the random variable $M_{\infty}$. The resulting equation is (9). Since this is the same as $\mathrm{SLE}_{\kappa}$ in $D$, and we know that (for $\kappa \leq 4) \mathrm{SLE}_{\kappa}$ in $D$ never leaves $D$, we conclude that with respect to the 
weighted measure, $T=\infty$. What this says is that for $\kappa \leq 8 / 3$, the probability measure on paths given by starting with $\mathrm{SLE}_{\kappa}$ from $x$ to $\infty$ in $\mathbb{H}$, and then taking the Radon-Nikodym derivative $M_{\infty} / M_{0}$, is the same as $\mathrm{SLE}_{\kappa}$ in $D$. Since $M_{0}=\Phi^{\prime}(x)^{b}$ and $M_{\infty}=Y_{\infty}$, we conclude that $\mathbf{E}\left[Y_{\infty}\right]=\Phi^{\prime}(x)^{b}$.

For $8 / 3<\kappa \leq 4$, we have $\lambda<0$ and hence $Y_{t}$ is not bounded. However, the same conclusions hold. The reason is as follows. For a fixed $\gamma:(0, \infty) \rightarrow \mathbb{H}$ parametrized as in (8) with $\gamma(t) \rightarrow \infty$ and $\operatorname{dist}(\gamma(0, \infty), \mathbb{H} \backslash D)>0$, standard conformal mapping estimates show that $Y_{t} \rightarrow Y_{\infty}<\infty$. In other words, the only problem that one can have is that $Y_{t}$ blows up as $\gamma(t) \rightarrow \mathbb{H} \backslash D$. If we consider a stopping time of the form $\tau_{n}=\inf \left\{t: Y_{t} \geq n\right\}$, then we know that $Y_{t \wedge \tau_{n}}$ is a martingale. If we use Girsanov to weight the paths by this martingale, the paths satisfy (91). We know (for $\kappa \leq 4$ ) that paths that are given by this SDE never reach $\mathbb{H} \backslash D$ and go to infinity. Hence, $M_{t}$ is actually a uniformly integrable martingale with $\mathbf{E}\left[M_{\infty}\right]=\mathbf{E}\left[M_{0}\right]=\Phi^{\prime}(x)^{b} \leq 1$.

As a prelude to the next section, we can see that we could define the $\mathrm{SLE}_{\kappa}$ measure in $D$ to be the measure on paths whose Radon-Nikodym derivative with respect to $\mathrm{SLE}_{\kappa}$ in $D$ is $Y_{\infty}$. This is a finite measure with total measure $\Phi^{\prime}(x)^{b}$. If we normalize to make this a probability measure, then the corresponding measure is that of $\mathrm{SLE}_{\kappa}$ in $D$ from $x$ to $\infty$.

\section{SLE measure}

\subsection{One path $(n=1)$}

The configurational approach is to view chordal $\mathrm{SLE}_{\kappa}$ as not just a probability measure on paths connecting two specified points on the boundary, but rather as a finite measure on paths that when normalized gives chordal $\mathrm{SLE}_{\kappa}$ as defined by Schramm. Recall that we restrict our consideration to $\kappa \leq 4$. Suppose $D$ is a simply connected domain and $z, w$ are distinct boundary points at which $\partial D$ is analytic. Then the $\mathrm{SLE}_{\kappa}$ measure on paths in $D$ from $z$ to $w$ is defined to be

$$
Q_{D, b}(z, w)=H_{D, b}(z, w) \mu_{D, b}^{\#}(z, w)
$$

where $\mu_{D, b}^{\#}(z, w)$ is the $\mathrm{SLE}_{\kappa}$ probability measure, and $H_{D, b}(z, w)=\left|Q_{D, b}(z, w)\right|$ is determined by the scaling rule (2) from the kernel

$$
H_{b}(x, y)=H_{\mathbb{H}, b}(x, y)=|y-x|^{-2 b}
$$

as in (5). The conformal covariance rule

$$
f \circ Q_{D, b}(z, w)=\left|f^{\prime}(z)\right|^{b}\left|f^{\prime}(w)\right|^{b} Q_{f(D), b}(f(z), f(w))
$$

follows immediately from the conformal invariance of $\mu_{D, b}^{\#}(z, w)$ and the scaling rule (2) for $H_{D, b}(x, y)$. An important property of the measure is the boundary perturbation rule.

Proposition 3.1 (Boundary Perturbation). Suppose $D \subset D^{\prime} \subsetneq \mathbb{C}$ are simply connected domains. Suppose that $z, w$ are distinct points of $\partial D ; \partial D$ is analytic in neighborhoods of $z$, 
$w$; and $\partial D, \partial D^{\prime}$ agree in neighborhoods of $z, w$. Then $Q_{D, b}(z, w)$ is absolutely continuous with respect to $Q_{D^{\prime}, b}(z, w)$ with Radon-Nikodym derivative

$$
Y_{D, D^{\prime}, b}(z, w)(\gamma)=1\{\gamma \subset D\} \exp \left\{-\lambda m\left(D^{\prime} ; \gamma, D^{\prime} \backslash D\right)\right\} .
$$

In particular, the Radon-Nikodym derivative is a conformal invariant, and moreover

$$
H_{D, b}(z, w) \leq H_{D^{\prime}, b}(z, w) .
$$

Proof. It suffices to prove this for $D^{\prime}=\mathbb{H}$ and this is discussed in Section 2.3.

Remark. If $f: D^{\prime} \rightarrow f\left(D^{\prime}\right)$ is a conformal transformation, then

$$
Y_{f(D), f\left(D^{\prime}\right), b}(f(z), f(w))(f \circ \gamma)=Y_{D, D^{\prime}, b}(z, w)(\gamma)
$$

It follows that the Radon-Nikodym derivative

$$
Y_{D, D^{\prime}, b}(z, w)=\frac{d Q_{D, b}(z, w)}{d Q_{D^{\prime}, b}(z, w)}
$$

makes sense even for non-smooth boundaries provided that $D \subset D^{\prime}$ and the boundaries agree near $z$ and $w$. If $f: D^{\prime} \rightarrow \mathbb{H}$ is a conformal transformation with $f(z)=0, f(w)=\infty$, then $f\left(D^{\prime} \backslash D\right)$ is a bounded subset of $\mathbb{H}$ bounded away from 0 .

\subsection{Multiple paths}

We will now define the measures $Q_{D, b, n}$ for positive integers $n$. Let $\mathbf{z}=\left(z_{1}, \ldots, z_{n}\right)$, w $=$ $\left(w_{1}, \ldots, w_{n}\right)$ denote $n$-tuples of distinct points in $\partial D$ ordered counterclockwise as in the introduction. Then $Q_{D, b, n}(\mathbf{z}, \mathbf{w})$, the $n$-path $\mathrm{SLE}_{\kappa}$ measure in $D$, is defined to be the measure that is absolutely continuous with respect to the product measure

$$
Q_{D, b}\left(z_{1}, w_{1}\right) \times \cdots \times Q_{D, b}\left(z_{n}, w_{n}\right)
$$

with Radon-Nikodym derivative $Y(\bar{\gamma})=Y_{D, b, \mathbf{z}, \mathbf{w}}\left(\gamma^{1}, \ldots, \gamma^{n}\right)$ given by

$$
Y(\bar{\gamma})=1\left\{\gamma^{k} \cap \gamma^{l}=\emptyset, 1 \leq k<l \leq n\right\} \exp \left\{-\lambda \sum_{k=1}^{n-1} m\left(D ; \gamma^{k}, \gamma^{k+1}\right)\right\}
$$

If $\lambda \geq 0$, it is immediate that $Q_{D, b, n}(\mathbf{z}, \mathbf{w})$ is a finite measure. As we will see after we prove the next proposition, this is also true for $\lambda \geq-1 / 2$; see (13).

Note that $Y$ is conformally invariant. Consequently, the measures $Q_{D, b, n}$ satisfy the conformal covariance relation (11). Other important properties are given in the next two propositions.

Proposition 3.2 (Cascade Relation). For $1 \leq j \leq n$, if

$$
\begin{gathered}
\mathbf{z}=\left(z_{1}, \ldots, z_{n}\right), \quad \mathbf{w}=\left(w_{1}, \ldots, w_{n}\right), \quad \hat{\gamma}=\left(\gamma^{1}, \ldots, \gamma^{j-1}, \gamma^{j+1}, \ldots, \gamma^{n}\right), \\
\hat{\mathbf{z}}=\left(z_{1}, \ldots, z_{j-1}, z_{j+1}, \ldots, z_{n}\right), \quad \hat{\mathbf{w}}=\left(w_{1}, \ldots, w_{j-1}, w_{j+1}, \ldots, w_{n}\right),
\end{gathered}
$$


then the marginal measure on $\hat{\gamma}$ in $Q_{D, b, n}(\mathbf{z}, \mathbf{w})$ is absolutely continuous with respect to $Q_{D, b, n-1}(\hat{\mathbf{z}}, \hat{\mathbf{w}})$ with Radon-Nikodym derivative $H_{\hat{D}, b}\left(z_{j}, w_{j}\right)$. Here $\hat{D}$ is the subdomain of $D \backslash \hat{\gamma}$ whose boundary includes $z_{j}, w_{j}$. (If $z_{j}, w_{j}$ are contained in the boundary of different components, then $H_{\hat{D}, b}\left(z_{j}, w_{j}\right)=0$.) Moreover, the conditional distribution of $\gamma^{j}$ given $\hat{\gamma}$ is that of $S L E_{\kappa}$ from $z_{j}$ to $w_{j}$ in $\hat{D}$.

Proof. Let $\hat{Y}(\hat{\gamma})=Y_{D, b, \hat{\mathbf{z}}, \hat{\mathbf{w}}}(\hat{\gamma})$ denote the density of $Q_{D, b, n-1}(\hat{\mathbf{z}}, \hat{\mathbf{w}})$ with respect to the appropriate product measure as defined in (12). Then

$$
Y=\hat{Y} 1\left\{\gamma^{l} \cap \gamma^{k}=\emptyset, 1 \leq l<k \leq n\right\} \exp \{-\lambda \hat{m}\}
$$

where

$$
\hat{m}=m\left(D ; \gamma^{j-1}, \gamma^{j}\right)+m\left(D ; \gamma^{j}, \gamma^{j+1}\right)-m\left(D ; \gamma^{j-1}, \gamma^{j+1}\right)
$$

Simple inclusion-exclusion shows that $\hat{m}$ is the measure of loops in $D$ that intersect both $\gamma^{j}$ and $D \backslash \hat{D}$. The result now follows from (12) and the discussion in Section 2.3.

Remark. Let $H_{D, b, n}(\mathbf{z}, \mathbf{w})=\left|Q_{D, b, n}(\mathbf{z}, \mathbf{w})\right|$. It follows from the proposition and (11), that

$$
H_{D, b, n}(\mathbf{z}, \mathbf{w}) \leq H_{D, b, n-1}(\hat{\mathbf{z}}, \hat{\mathbf{w}}) H_{D, b}\left(z_{j}, w_{j}\right) .
$$

By iterating, we get

$$
H_{D, b, n}(\mathbf{z}, \mathbf{w}) \leq \prod_{j=1}^{n} H_{D, b}\left(z_{j}, w_{j}\right)
$$

so that we conclude $Q_{D, b, n}(\mathbf{z}, \mathbf{w})$ is a finite measure for all $b \geq 1 / 4$, or equivalently, all $\lambda \geq-1 / 2$.

Proposition 3.3 (Boundary Perturbation). Suppose $D \subset D^{\prime} \subsetneq \mathbb{C}$ are simply connected domains. Then $Q_{D, b, n}(\mathbf{z}, \mathbf{w})$ is absolutely continuous with respect to $Q_{D^{\prime}, b, n}(\mathbf{z}, \mathbf{w})$ with RadonNikodym derivative

$$
Y_{D, D^{\prime}, b, n}(\mathbf{z}, \mathbf{w})(\bar{\gamma})=1\left\{\gamma^{j} \subset D, j=1, \ldots, n\right\} \exp \left\{-\lambda m\left(D^{\prime} ; \gamma^{1} \cup \cdots \cup \gamma^{n}, D^{\prime} \backslash D\right)\right\} .
$$

In particular, the Radon-Nikodym derivative is a conformal invariant.

Proof. It follows from Proposition 3.1 that the Radon-Nikodym derivative of

$$
Q_{D, b}\left(z_{1}, w_{1}\right) \times \cdots \times Q_{D, b}\left(z_{n}, w_{n}\right)
$$

with respect to

$$
Q_{D^{\prime}, b}\left(z_{1}, w_{1}\right) \times \cdots \times Q_{D^{\prime}, b}\left(z_{n}, w_{n}\right)
$$

is

$$
1\left\{\gamma^{j} \subset D, j=1, \ldots, n\right\} \exp \left\{-\lambda \sum_{k=1}^{n} m\left(D^{\prime} ; \gamma^{k}, D^{\prime} \backslash D\right)\right\} .
$$

Hence by (12), $Y_{D, D^{\prime}, b, n}(\mathbf{z}, \mathbf{w})(\bar{\gamma})$ equals this quantity times

$$
\exp \left\{-\lambda \sum_{k=1}^{n-1}\left[m\left(D ; \gamma^{k}, \gamma^{k+1}\right)-m\left(D^{\prime} ; \gamma^{k}, \gamma^{k+1}\right)\right]\right\} .
$$


But $m\left(D^{\prime} ; \gamma^{k}, \gamma^{k+1}\right)-m\left(D ; \gamma^{k}, \gamma^{k+1}\right)$ denotes the measure of the set of loops in $D^{\prime}$ that intersect all three of $\gamma^{k}, \gamma^{k+1}$, and $D^{\prime} \backslash D$. Therefore, for $k=1, \ldots, n-1$,

$$
m\left(D^{\prime} ; \gamma^{k}, D^{\prime} \backslash D\right)-\left[m\left(D^{\prime} ; \gamma^{k}, \gamma^{k+1}\right)-m\left(D ; \gamma^{k}, \gamma^{k+1}\right)\right]
$$

denotes the measure of the set of loops in $D$ that intersect $\gamma^{k}$ and $D^{\prime} \backslash D$ but do not intersect $\gamma^{k+1}$. For topological reasons, they cannot possibly intersect $\gamma^{l}$ for $l \geq k+1$. For any loop in $D^{\prime}$ that intersects both $\gamma^{1} \cup \cdots \cup \gamma^{n}$, there is a largest $k$ such that the loop intersects $\gamma^{k}$ but not $\gamma^{k+1}, \ldots, \gamma^{n}$. This is accounted for in the $k$ th term of the sum and in no other.

Recall that $H_{D, b, n}$ satisfies the scaling rule

$$
H_{D, b, n}(\mathbf{z}, \mathbf{w})=\left|f^{\prime}(\mathbf{z})\right|^{b}\left|f^{\prime}(\mathbf{w})\right|^{b} H_{f(D), b, n}(f(\mathbf{z}), f(\mathbf{w})) .
$$

It also satisfies the following cascade relation. Suppose $\mathbf{z}=\left(z_{1}, \hat{\mathbf{z}}\right), \mathbf{w}=\left(w_{1}, \hat{\mathbf{w}}\right)$ and all the points of $\hat{\mathbf{z}}, \hat{\mathbf{w}}$ lie on the same arc connecting $z_{1}$ and $w_{1}$ on $\partial D$. Then,

$$
H_{D, b, n}(\mathbf{z}, \mathbf{w})=Q_{D, b}\left(z_{1}, w_{1}\right)\left[H_{D(\gamma), b, n-1}(\hat{\mathbf{z}}, \hat{\mathbf{w}})\right]
$$

where $D(\gamma)$ is the connected component of $D \backslash \gamma$ whose boundary contains $\hat{\mathbf{z}}, \hat{\mathbf{w}}$ and we use the shorthand notation $\mu[f]=\int f d \mu$. If $f: D \rightarrow \mathbb{H}$ is a conformal transformation with $f\left(z_{1}\right)=0, f\left(w_{1}\right)=\infty$, and $\hat{\mathbf{z}}, \hat{\mathbf{w}}$ are sent to the positive real axis, then

$$
H_{D, b, n}(\mathbf{z}, \mathbf{w})=\left|Q_{D, b}\left(z_{1}, w_{1}\right)\right|\left|f^{\prime}(\hat{\mathbf{z}})\right|^{b}\left|f^{\prime}(\hat{\mathbf{w}})\right|^{b} \mathbf{E}\left[H_{(\mathbb{H} \backslash \gamma)_{+}, b, n-1}(f(\hat{\mathbf{z}}), f(\hat{\mathbf{w}}))\right],
$$

where in this case the expectation is over $\mathrm{SLE}_{\kappa}$ from 0 to $\infty$ in $\mathbb{H}$ and $(\mathbb{H} \backslash \gamma)_{+}$denotes the component of $\mathbb{H} \backslash \gamma$ to the right of $\gamma$. This shows that $Q_{D, b, n}$ for all $n$ can be computed, at least in principle, from computations of $\mathrm{SLE}_{\kappa}$ in $\mathbb{H}$.

We will also define the partition function

$$
\tilde{H}_{D, b, n}(\mathbf{z}, \mathbf{w})=\frac{H_{D, b, n}(\mathbf{z}, \mathbf{w})}{H_{D, b}\left(z_{1}, w_{1}\right) \cdots H_{D, b}\left(z_{n}, w_{n}\right)} .
$$

Note that this is a conformal invariant, i.e.,

$$
\tilde{H}_{D, b, n}(\mathbf{z}, \mathbf{w})=\tilde{H}_{f(D), b, n}(f(\mathbf{z}), f(\mathbf{w})) .
$$

In particular $H_{D, b, n}$, and $\tilde{H}_{D, b, n}$ are determined by the values of

$$
H_{b, n}(\mathbf{x}, \mathbf{y})=H_{\mathbb{H}, b, n}(\mathbf{x}, \mathbf{y}),
$$

for $\mathbf{x}, \mathbf{y} \in \mathbb{R}^{n}$. We also write $\tilde{H}_{b, n}$ for $\tilde{H}_{\mathbb{H}, b, n}$.

Remark. One case that is known is $\mathrm{SLE}_{2}$, corresponding to loop-erased walk $(a=b=1)$, in which case

$$
H_{1, n}(\mathbf{x}, \mathbf{y})=\operatorname{det}\left[\left(y_{k}-x_{j}\right)^{-2}\right]_{1 \leq j, k \leq n} .
$$

This is a consequence of Fomin's identity and the $n=1$ case. We describe this in more detail in Section 5 . 


$$
\begin{aligned}
& \text { If } 0<x_{1}<\cdots<x_{n}<y_{n}<\cdots<y_{1}<\infty \text {, we let } \\
& \qquad \tilde{H}_{b, n}^{*}(\mathbf{x}, \mathbf{y})=\tilde{H}_{b, n+1}((0, \mathbf{x}),(\infty, \mathbf{y}))=\lim _{w \rightarrow \infty} \tilde{H}_{b, n+1}((0, \mathbf{x}),(w, \mathbf{y})), \\
& H_{b, n}^{*}(\mathbf{x}, \mathbf{y})=\left[\prod_{j=1}^{n} H_{b, 1}\left(x_{j}, y_{j}\right)\right] \tilde{H}_{b, n}^{*}(\mathbf{x}, \mathbf{y})=\lim _{w \rightarrow \infty} w^{2 b} H_{b, n+1}((0, \mathbf{x}),(w, \mathbf{y})) .
\end{aligned}
$$

These functions satisfy the following scaling rules for $r>0$ :

$$
\tilde{H}_{b, n}^{*}(r \mathbf{x}, r \mathbf{y})=\tilde{H}_{b, n}^{*}(\mathbf{x}, \mathbf{y}), \quad H_{b, n}^{*}(r \mathbf{x}, r \mathbf{y})=r^{-2 b n} H_{b, n}^{*}(\mathbf{x}, \mathbf{y}) .
$$

\subsection{The partition function for two paths}

In this section we calculate $H_{b, 1}^{*}(x, y)=(y-x)^{-2 b} \tilde{H}_{b, 1}^{*}(x, y)=(y-x)^{-2 b} \tilde{H}_{b, 2}((0, x),(\infty, y))$ for $0<x<y<\infty$. The formula in the following proposition has appeared previously; see, for example, [3]. As usual, we will write just $H^{*}(x, y), \tilde{H}^{*}(x, y)$ for $H_{b, 1}^{*}(x, y), \tilde{H}_{b, 1}^{*}(x, y)$, respectively. We will use this result in Section 5.4 to derive the scaling limit of Fomin's identity.

Proposition 3.4. If $b \geq 1 / 4$, then

$$
H_{b, 1}^{*}(x, y)=(y-x)^{-2 b} \frac{\Gamma(2 a) \Gamma(6 a-1)}{\Gamma(4 a) \Gamma(4 a-1)}(x / y)^{a} F(2 a, 1-2 a, 4 a ; x / y)=\frac{\phi_{b}(x / y)}{(y-x)^{2 b}},
$$

where $F$ denotes the hypergeometric function and $a=(2 b+1) / 3$.

Proof. By the scaling rule (16), we know that $\tilde{H}^{*}(x, y)=\phi(x / y)$ for some function $\phi=\phi_{b}$ of one variable. We will parametrize $\mathrm{SLE}_{\kappa}$ so that it satisfies (7) where the driving function $U_{t}=-B_{t}$ is a standard one-dimensional Brownian motion. Let $0<x<y$, and let $X_{t}=$ $g_{t}(x)+B_{t}, Y_{t}=g_{t}(y)+B_{t}$ so that

$$
d X_{t}=\frac{a}{X_{t}} d t+d B_{t}, \quad d Y_{t}=\frac{a}{Y_{t}} d t+d B_{t}
$$

as in the proof of Proposition 2.1. Let $(\mathbb{H} \backslash \gamma)_{+}$denote the component of $\mathbb{H} \backslash \gamma$ whose boundary includes $x, y$. If $g:(\mathbb{H} \backslash \gamma)_{+} \rightarrow \mathbb{H}$ is a conformal transformation with $g(\infty)=\infty$, then

$$
H_{(\mathbb{H} \backslash \gamma)_{+}, b}(x, y)=\frac{g^{\prime}(x)^{b} g^{\prime}(y)^{b}}{[g(y)-g(x)]^{2 b}}
$$

as a consequence of (21) and (15). Using this we see that if

$$
J_{t}=\frac{g_{t}^{\prime}(x)^{b} g_{t}^{\prime}(y)^{b}}{\left[g_{t}(y)-g_{t}(x)\right]^{2 b}} \quad \text { and } \quad J_{\infty}=\lim _{t \rightarrow \infty} J_{t}
$$

then from (14) and (15)

$$
\tilde{H}^{*}(x, y)=(y-x)^{2 b} \mathbf{E}^{x, y}\left[J_{\infty}\right]
$$


so that $H^{*}(x, y)=\mathbf{E}^{x, y}\left[J_{\infty}\right]$. We will now give the differential equation for $\tilde{H}^{*}(x, y)$. Since

$$
\begin{gathered}
\partial_{t}\left[\log g_{t}^{\prime}(x)\right]=-\frac{a}{X_{t}^{2}}, \quad \partial_{t}\left[\log g_{t}^{\prime}(y)\right]=-\frac{a}{Y_{t}^{2}}, \quad \text { and } \\
\partial_{t}\left[\log \left(g_{t}(y)-g_{t}(x)\right)\right]=\frac{1}{Y_{t}-X_{t}}\left[\frac{a}{Y_{t}}-\frac{a}{X_{t}}\right]=-\frac{a}{X_{t} Y_{t}},
\end{gathered}
$$

we see that

$$
J_{t}=\exp \left\{\log J_{t}\right\}=J_{0} \exp \left\{\int_{0}^{t} \partial_{s}\left[\log J_{s}\right] d s\right\}=\frac{1}{(y-x)^{2 b}} \exp \left\{-a b \int_{0}^{t}\left(\frac{1}{X_{s}}-\frac{1}{Y_{s}}\right)^{2} d s\right\} .
$$

Hence, (18) gives

$$
\tilde{H}^{*}(x, y)=\mathbf{E}^{x, y}\left[\exp \left\{-a b \int_{0}^{\infty}\left(\frac{1}{X_{s}}-\frac{1}{Y_{s}}\right)^{2} d s\right\}\right]
$$

It now follows from the (usual) Markov property that $J_{t} \tilde{H}^{*}\left(X_{t}, Y_{t}\right)$ is a martingale. That is, if $M_{t}=\mathbf{E}^{x, y}\left[J_{\infty} \mid \mathcal{F}_{t}\right]$ so that $M_{t}$ is a martingale, then

$$
\begin{aligned}
M_{t} & =\mathbf{E}^{x, y}\left[\exp \left\{-a b \int_{0}^{\infty}\left(\frac{1}{X_{s}}-\frac{1}{Y_{s}}\right)^{2} d s\right\} \mid \mathcal{F}_{t}\right] \\
& =\exp \left\{-a b \int_{0}^{t}\left(\frac{1}{X_{s}}-\frac{1}{Y_{s}}\right)^{2} d s\right\} \mathbf{E}^{x, y}\left[\exp \left\{-a b \int_{t}^{\infty}\left(\frac{1}{X_{s}}-\frac{1}{Y_{s}}\right)^{2} d s\right\} \mid \mathcal{F}_{t}\right] \\
& =J_{t} \tilde{H}^{*}\left(X_{t}, Y_{t}\right) .
\end{aligned}
$$

Itô's formula now implies that

$$
-a b\left(\frac{1}{x}-\frac{1}{y}\right)^{2} \tilde{H}^{*}+\frac{a}{x} \partial_{x} \tilde{H}^{*}+\frac{a}{y} \partial_{y} \tilde{H}^{*}+\frac{1}{2} \partial_{x x} \tilde{H}^{*}+\frac{1}{2} \partial_{y y} \tilde{H}^{*}+\partial_{x y} \tilde{H}^{*}=0 .
$$

Since $\tilde{H}^{*}(x, y)=\phi(x / y)$, we find

$$
\begin{gathered}
\partial_{x} \tilde{H}^{*}=y^{-1} \phi^{\prime}(x / y), \quad \partial_{y} \tilde{H}^{*}=-x y^{-2} \phi^{\prime}(x / y), \quad \partial_{x x} \tilde{H}^{*}=y^{-2} \phi^{\prime \prime}(x / y), \\
\partial_{y y} \tilde{H}^{*}=2 x y^{-3} \phi^{\prime}(x / y)+x^{2} y^{-4} \phi^{\prime \prime}(x / y), \quad \partial_{x y} \tilde{H}^{*}=-y^{-2} \phi^{\prime}(x / y)-x y^{-3} \phi^{\prime \prime}(x / y),
\end{gathered}
$$

so that after substituting into (19), multiplying by $y^{2}$, letting $u=x / y$, combining terms, and recalling that $b=(3 a-1) / 2$, we have

$$
u^{2}(1-u)^{2} \phi^{\prime \prime}(u)+2 u\left(a-u+(1-a) u^{2}\right) \phi^{\prime}(u)-a(3 a-1)(1-u)^{2} \phi(u)=0 .
$$

Note that $0<u<1$, and let $\psi(u)=u^{-a}(1-u)^{1-4 a} \phi(u)$ so that (20) implies

$$
u(1-u) \psi^{\prime \prime}(u)+(4 a-8 a u) \psi^{\prime}(u)-2 a(6 a-1) \psi(u)=0 .
$$


We see that (21) is the well-known hypergeometric differential equation (see equation (9.5.5) of [15]) which has two linearly independent solution; hence

$$
\psi(u)=C_{1} F(2 a, 6 a-1,4 a ; u)+C_{2} u^{1-4 a} F(1-2 a, 2 a, 2-4 a ; u)
$$

where $C_{1}$ and $C_{2}$ are arbitrary constants. Equation (9.5.3) of [15] gives $F(2 a, 6 a-1,4 a ; u)=$ $(1-u)^{1-4 a} F(2 a, 1-2 a, 4 a ; u)$ so that the general solution of (20) is therefore

$$
\phi(u)=C_{1} u^{a} F(2 a, 1-2 a, 4 a ; u)+C_{2} u^{1-3 a}(1-u)^{4 a-1} F(2 a, 1-2 a, 2-4 a ; u) .
$$

Since $\phi(u) \rightarrow 0$ as $u \rightarrow 0+$, and $\phi(u) \rightarrow 1$ as $u \rightarrow 1-$, we find $C_{2}=0$ and

$$
C_{1}^{-1}=F(2 a, 1-2 a, 4 a ; 1)=\lim _{u \rightarrow 1-} F(2 a, 1-2 a, 4 a ; u)=\frac{\Gamma(4 a) \Gamma(4 a-1)}{\Gamma(2 a) \Gamma(6 a-1)},
$$

so that

$$
\phi(u)=\phi_{b}(u)=\frac{\Gamma(2 a) \Gamma(6 a-1)}{\Gamma(4 a) \Gamma(4 a-1)} u^{a} F(2 a, 1-2 a, 4 a ; u) .
$$

Remark. If $x>0$ is fixed, then

$$
\lim _{y \rightarrow \infty} y^{a+2 b} H_{b, 1}^{*}(x, y)=\frac{\Gamma(2 a) \Gamma(6 a-1)}{\Gamma(4 a) \Gamma(4 a-1)} x^{a} .
$$

Remark. If $\tilde{H}_{b, 2}=\tilde{H}_{\mathbb{H}, b, 2}$ is as defined in (15), then this proposition implies for $x_{1}<x_{2}<$ $y_{2}<y_{1}$ that

$$
\tilde{H}_{b, 2}\left(x_{1}, x_{2}, y_{1}, y_{2}\right)=\phi_{b}(1-q)
$$

where

$$
q=q\left(x_{1}, x_{2}, y_{2}, y_{1}\right)=\frac{\left(y_{1}-x_{1}\right)\left(y_{2}-x_{2}\right)}{\left(y_{1}-x_{2}\right)\left(y_{2}-x_{1}\right)},
$$

denotes the cross-ratio. As pointed out in [3], if the quantity on the left of (23) is to be a conformal invariant, it should be a function of $q$. If $x_{1}=0, x_{2}=x, y_{2}=y, y_{1}=\infty$, then $q=1-(x / y)$ as expected.

Remark. If $1-2 a=-k$ where $k$ is a non-negative integer, then $F(2 a, 1-2 a, 4 a ; x / y)$ is a $k$ th degree polynomial. These values correspond to

$$
b=\frac{3 k+1}{4} .
$$

In particular, $\phi_{1 / 4}(u)=\sqrt{u}$ and $\phi_{1}(u)=2 u-u^{2}=1-(1-u)^{2}$. Several other special cases of (17) are listed in the following table.

\begin{tabular}{|c|c|c|c|}
\hline$a=$ & $b=$ & $\kappa=$ & $H_{b, 1}^{*}(x, y)=$ \\
\hline \hline 2 & $5 / 2$ & 1 & $(y-x)^{-5}(x / y)^{2}\left[6-9(x / y)+5(x / y)^{2}-(x / y)^{3}\right]$ \\
\hline $3 / 2$ & $7 / 4$ & $4 / 3$ & $(y-x)^{-7 / 2}(x / y)^{3 / 2}\left[(7 / 2)-(7 / 2)(x / y)+(x / y)^{2}\right]$ \\
\hline 1 & 1 & 2 & $(y-x)^{-2}(x / y)[2-(x / y)]$ \\
\hline $1 / 2$ & $1 / 4$ & 4 & $(y-x)^{-1 / 2}(x / y)^{1 / 2}$ \\
\hline
\end{tabular}


Remark. Our argument is essentially the same as the calculation of Cardy's formula for $\mathrm{SLE}_{\kappa}$. An examination of the proof will show that the hypergeometric solution is valid for $a>1 / 4$, or, equivalently, $\kappa<8$. The only reason we restrict to $b \geq 1 / 4$ in the proposition is to have $\kappa \leq 4$. In fact, if $\kappa=6$ (corresponding to $b=0$, or $a=1 / 3$ ) then we recover Cardy's formula, namely

$$
\phi_{0}(u)=\frac{\Gamma(2 / 3)}{\Gamma(4 / 3) \Gamma(1 / 3)} u^{1 / 3} F(2 / 3,1 / 3,4 / 3 ; u) .
$$

A proof for the $b=0$ case only may be found in either [2] or [8, Proposition 6.33].

\section{Multiple SLEs}

As was shown in Section 3.2, the definition of $Q_{D, b, n}(\mathbf{z}, \mathbf{w})$ is fairly straightforward. Since $H_{D, b, n}(\mathbf{z}, \mathbf{w})=\left|Q_{D, b, n}(\mathbf{z}, \mathbf{w})\right|$, we can write

$$
Q_{D, b, n}(\mathbf{z}, \mathbf{w})=H_{D, b, n}(\mathbf{z}, \mathbf{w}) \mu_{D, b, n}^{\#}(\mathbf{z}, \mathbf{w}),
$$

for some probability measure $\mu_{D, b, n}^{\#}(\mathbf{z}, \mathbf{w})$ on $n$-tuples of paths that can be called $\mathrm{SLE}_{\kappa}$ from $\mathbf{z}$ to $\mathbf{w}$ in $D$. This measure will be absolutely continuous with respect to the product measure given by taking $n$ independent $\mathrm{SLE}_{\kappa}$ paths $\gamma^{1}, \ldots, \gamma^{n}$ where $\gamma^{j}$ goes from $z_{j}$ to $w_{j}$. In the present section, we use Girsanov's theorem to give the SDE.

Suppose $G\left(t, x_{0}, x_{1}, \ldots, x_{n}\right)$ is a smooth, non-negative function (at least on the set $\left\{x_{j} \neq\right.$ $\left.\left.x_{k}: 0 \leq j<k \leq n\right\}\right)$, and suppose $\gamma:(0, \infty) \rightarrow \mathbb{H}$ is an $\mathrm{SLE}_{\kappa}$ curve with corresponding maps $g_{t}$ parametrized as in (7). We allow the function $G\left(t, x_{0}, x_{1}, \ldots, x_{n}\right)$ to be a random function adapted to the Brownian filtration, but we assume that with probability one $G$ is $C^{1}$ in $t$. Let $X_{t}^{j}=g_{t}\left(x_{j}\right)$ where $W_{t}$ is a standard Brownian motion, and note that

$$
\dot{X}_{t}^{j}=\frac{a}{X_{t}^{j}-W_{t}}
$$

If $J_{t}=G\left(t, W_{t}, X_{t}^{1}, \ldots, X_{t}^{n}\right)$, then Itô's formula gives

$$
d J_{t}=J_{t}\left[R_{t} d t+\frac{\partial_{0} G\left(t, W_{t}, \bar{X}_{t}\right)}{G\left(t, W_{t}, \bar{X}_{t}\right)} d W_{t}\right]
$$

where

$$
R_{t}=G\left(t, W_{t}, \bar{X}_{t}\right)^{-1}\left[\dot{G}\left(t, W_{t}, \bar{X}_{t}\right)+\frac{1}{2} \partial_{00} G\left(t, W_{t}, \bar{X}_{t}\right)+a \sum_{j=1}^{n} \frac{\partial_{j} G\left(t, W_{t}, \bar{X}_{t}\right)}{X_{t}^{j}-W_{t}}\right]
$$

with $\bar{X}_{t}=\left(X_{t}^{1}, \ldots, X_{t}^{n}\right)$ and $\partial_{j}=\partial_{x_{j}}, \partial_{00}=\partial_{x_{0} x_{0}}$. If

$$
M_{t}=\exp \left\{-\int_{0}^{t} R_{s} d s\right\} J_{t}
$$


then $M_{t}$ is a local martingale satisfying

$$
d M_{t}=M_{t} \frac{\partial_{0} G\left(t, W_{t}, \bar{X}_{t}\right)}{G\left(t, W_{t}, \bar{X}_{t}\right)} d W_{t}
$$

If $M_{t}$ is a martingale, Girsanov's theorem says that if we weight the paths by $M_{t}$ then this is the same as if $W_{t}$ satisfied

$$
d W_{t}=\frac{\partial_{0} G\left(t, W_{t}, \bar{X}_{t}\right)}{G\left(t, W_{t}, \bar{X}_{t}\right)} d t+d B_{t},
$$

for a standard Brownian motion $B_{t}$. Hence an $\mathrm{SLE}_{\kappa}$ weighted by $G$ is the same as a solution to the chordal Loewner equation (7) where the driving function $W_{t}$ satisfies (24). Even if $M_{t}$ is not a martingale, we can get a similar expression for a stopped process $M_{t \wedge \tau}$ if the stopped process is a continuous martingale.

\subsection{SLE in a domain $D$}

Suppose that $D \subset \mathbb{H}$ is a simply connected domain with $\mathbb{H} \backslash D$ bounded and bounded away from zero. Let $D_{t}=g_{t}(D)$ and let $\Phi_{t}$ be a conformal transformation of $D_{t}$ onto $\mathbb{H}$ with $\Phi_{t}(\infty)=\infty, \Phi_{t}^{\prime}(\infty)=1$. Let $G(t, x)=\Phi_{t}^{\prime}(x)^{b}$. Then if we weight the paths of $\mathrm{SLE}_{\kappa}$ by this function, the paths satisfy

$$
d W_{t}=b \frac{\Phi_{t}^{\prime \prime}\left(W_{t}\right)}{\Phi_{t}^{\prime}\left(W_{t}\right)} d t+d B_{t}
$$

This is the same equation as given in Section 2.3 for $\mathrm{SLE}_{\kappa}$ in $D$ when one takes the conformal image of $\mathrm{SLE}_{\kappa}$ from $\mathbb{H}$ to $D$. In other words, another way of viewing $\mathrm{SLE}_{\kappa}$ in $D$ is as $\mathrm{SLE}_{\kappa}$ weighted by $\Phi_{t}\left(W_{t}\right)^{b}$.

A similar example is $\mathrm{SLE}_{\kappa}$ from 0 to $y$ where $0<y<\infty$. This measure on paths is absolutely continuous with respect to $\mathrm{SLE}_{\kappa}$ from 0 to $\infty$ provided that the paths are stopped at a stopping time smaller than the first visit to $y$. At that point the paths become singular. (Although this is somewhat imprecise, it is not too difficult to make it precise.) The equation (25) can be applied to see how the process evolves. If $t$ is smaller than the hitting time of $y$, we define $g_{t}$ as before and let $X_{t}=g_{t}(y)$. Then, $\Phi_{t}(z)=\left(z-X_{t}\right)^{-1}$ is a conformal map taking $g_{t}(\mathbb{H} \backslash \gamma(0, t])\left(=\mathbb{H}\right.$ in this case) to $\mathbb{H}$, mapping $X_{t}$ to $\infty$. Using (25), we get

$$
d W_{t}=\frac{2 b}{X_{t}-W_{t}} d t+d B_{t}, \quad d X_{t}=\frac{a}{X_{t}-W_{t}} d t
$$

This is an example of an $\operatorname{SLE}(\kappa, \rho)$ process as introduced in [10]. Note that

$$
d\left(W_{t}-X_{t}\right)=\frac{(1-4 b) / 3}{W_{t}-X_{t}} d t+d B_{t},
$$

so comparison with a Bessel process shows that the path has finite lifetime. (Recall that we are parametrizing the curve by its half-plane capacity - if $\gamma$ is a simple curve from 0 to $y$, then the path has finite capacity.) We can derive the same process by taking $\mathrm{SLE}_{\kappa}$ from 0 to $\infty$ and weighting the paths by $G\left(t, W_{t}, X_{t}\right)=H_{b}\left(W_{t}, X_{t}\right)=\left(X_{t}-W_{t}\right)^{-2 b}$. We can see this by comparing (24) and the first equation in (26). 


\subsection{Two $\mathrm{SLE}_{\kappa}$ paths}

Consider the case of the $\mathrm{SLE}_{\kappa}$ measure corresponding to two mutually avoiding $\mathrm{SLE}_{\kappa}$ paths; one from 0 to $\infty$ and the other from $x_{1}$ to $x_{2}$. This corresponds to choosing

$$
G\left(t, x_{0}, x_{1}, x_{2}\right)=H^{*}\left(x_{1}-x_{0}, x_{2}-x_{0}\right)=\left(x_{2}-x_{1}\right)^{-2 b} \phi_{b}\left(1-\frac{x_{2}-x_{1}}{x_{2}-x_{0}}\right)
$$

where $\phi_{b}$ is as in Proposition 3.4. (Recall that $H^{*}(x, y)=H_{\mathbb{H}, b, 1}^{*}(x, y)$.) Then,

$$
\frac{\partial_{0} G\left(t, x_{0}, x_{1}, x_{2}\right)}{G\left(t, x_{0}, x_{1}, x_{2}\right)}=-\frac{1}{x_{2}-x_{0}} \frac{q \phi_{b}^{\prime}(1-q)}{\phi_{b}(1-q)}=-\frac{q}{x_{2}-x_{0}}\left[\log \phi_{b}\right]^{\prime}(1-q),
$$

where $\partial_{0}=\partial_{x_{0}}$ as before and $q=\left(x_{2}-x_{1}\right) /\left(x_{2}-x_{0}\right)$ denotes the appropriate cross-ratio. This is the marginal distribution of the $\mathrm{SLE}_{\kappa}$ path from 0 to $\infty$ given by the measure $\mu_{b, 2}^{\#}\left(\left(0, x_{1}\right),\left(x_{2}, \infty\right)\right)$. Two examples are as follows.

- If $b=1$ (equivalently, $\kappa=2$ ), we have $\phi_{1}(u)=2 u-u^{2}$ and hence

$$
\frac{\partial_{0} G\left(t, x_{0}, x_{1}, x_{2}\right)}{G\left(t, x_{0}, x_{1}, x_{2}\right)}=-\frac{2}{x_{2}-x_{0}} \frac{q^{2}}{1-q^{2}} \text {. }
$$

- If $b=1 / 4$ (equivalently, $\kappa=4$ ), then $\phi_{1 / 4}(u)=\sqrt{u}$ and hence

$$
\frac{\partial_{0} G\left(t, x_{0}, x_{1}, x_{2}\right)}{G\left(t, x_{0}, x_{1}, x_{2}\right)}=-\frac{1}{2\left(x_{2}-x_{0}\right)} \frac{q}{1-q} .
$$

If we let $x_{2} \rightarrow \infty$, we can consider two mutually avoiding SLE $_{\kappa}$ paths both going to infinity. Here we use the function (see (22)

$$
G\left(t, x_{0}, x_{1}\right)=\left(x_{1}-x_{0}\right)^{a} .
$$

In this case,

$$
\frac{\partial_{0} G\left(t, x_{0}, x_{1}\right)}{G\left(t, x_{0}, x_{1}\right)}=-\frac{a}{x_{1}-x_{0}} .
$$

From this we see that if we take a pair mutually avoiding $\mathrm{SLE}_{\kappa}$ paths starting at $x_{0}, x_{1}$, respectively, then the measure on the path started at $x_{0}$ is obtained by solving the usual chordal Loewner equation with driving function $U_{t}$ satisfying

$$
d U_{t}=\frac{a}{U_{t}-V_{t}} d t+d B_{t}
$$

where $V_{t}=g_{t}\left(x_{1}\right)$ satisfies

$$
d V_{t}=\frac{a}{V_{t}-U_{t}} d t
$$

This is an example of a $\operatorname{SLE}(\kappa, \rho)$ process in [10]. Note that $Z_{t}=U_{t}-V_{t}$ satisfies

$$
d Z_{t}=\frac{2 a}{Z_{t}} d t+d B_{t}
$$




\subsubsection{Growing both paths simultaneously}

It is possible to write down the equation for two mutually avoiding SLE paths growing simultaneously. We can write the corresponding conformal transformation $g_{t}$ as the solution to the Loewner equation

$$
\dot{g}_{t}(z)=\frac{a}{g_{t}(z)-U_{t}^{1}}+\frac{a}{g_{t}(z)-U_{t}^{2}},
$$

for appropriate driving functions $U_{t}^{1}, U_{t}^{2}$. The parametrization of $g_{t}$ is such that

$$
g_{t}(z)=z+\frac{2 a}{z}+O\left(|z|^{-2}\right), \quad z \rightarrow \infty .
$$

To find the equation of $U_{t}^{j}$ we start by taking them to satisfy

$$
d U_{t}^{1}=\frac{a}{U_{t}^{1}-U_{t}^{2}} d t+d W_{t}^{1} \text { and } d U_{t}^{2}=\frac{a}{U_{t}^{2}-U_{t}^{1}} d t+d W_{t}^{2},
$$

where $W_{t}^{1}, W_{t}^{2}$ are independent standard Brownian motions.

Assume that $U_{0}^{1}=x_{0}$ and $U_{0}^{2}=x_{1}$. If $G\left(t, x_{0}, x_{1}\right)=\left(x_{1}-x_{0}\right)^{a}$, then $G\left(t, U_{t}^{1}, U_{t}^{2}\right)$ satisfies

$$
\begin{aligned}
\frac{d G\left(t, U_{t}^{1}, U_{t}^{2}\right)}{G\left(t, U_{t}^{1}, U_{t}^{2}\right)} & =\frac{a(a-1)}{\left(U_{t}^{2}-U_{t}^{1}\right)^{2}} d t+\frac{a}{U_{t}^{1}-U_{t}^{2}} d U_{t}^{1}+\frac{a}{U_{t}^{2}-U_{t}^{1}} d U_{t}^{2} \\
& =\frac{a(3 a-1)}{\left(U_{t}^{2}-U_{t}^{1}\right)^{2}} d t+\frac{a}{U_{t}^{1}-U_{t}^{2}} d W_{t}^{1}+\frac{a}{U_{t}^{2}-U_{t}^{1}} d W_{t}^{2}
\end{aligned}
$$

Again,

$$
M_{t}=\exp \left\{-a(3 a-1) \int_{0}^{t}\left(U_{s}^{2}-U_{s}^{1}\right)^{-2} d s\right\} G\left(t, U_{t}^{1}, U_{t}^{2}\right)
$$

is a local martingale satisfying

$$
d M_{t}=M_{t}\left[\frac{a}{U_{t}^{1}-U_{t}^{2}} d W_{t}^{1}+\frac{a}{U_{t}^{2}-U_{t}^{1}} d W_{t}^{2}\right] .
$$

Compare this with [8, Example 1.18]. If we weight the paths by $M_{t}$, the effective equation on the $W_{t}^{j}$ is

$$
d W_{t}^{j}=\frac{a}{U_{t}^{j}-U_{t}^{3-j}} d t+d B_{t}^{j}
$$

where $B_{t}^{1}, B_{t}^{2}$ denote independent standard Brownian motions, and the effective equation on the $U_{t}^{j}$ becomes

$$
d U_{t}^{j}=\frac{2 a}{U_{t}^{j}-U_{t}^{3-j}} d t+d B_{t}^{j} .
$$

Note that if $Z_{t}=U_{t / 2}^{2}-U_{t / 2}^{1}, B_{t}=B_{t / 2}^{2}-B_{t / 2}^{1}$, then $Z_{t}, B_{t}$ satisfy (28). 


\subsection{Multiple SLE $_{\kappa}$}

Suppose $x_{1}<\cdots<x_{n}<y_{n}<\cdots<y_{1}$. Then the measure $\mu_{\mathbb{H}, b, n}^{\#}(\mathbf{x}, \mathbf{y})$ can be described by using the function

$$
G\left(t, x_{1}, \ldots, x_{n}, y_{1}, \ldots, y_{n}\right)=H_{b, n}(\mathbf{x}, \mathbf{y}) .
$$

We can use this to describe the marginal distribution for a particular path. For example, suppose we are interested in the first path. Then it acts like $\mathrm{SLE}_{\kappa}$ weighted locally by $G\left(t, W_{t}, X_{t}^{2}, \ldots, X_{t}^{n}, Y_{t}^{1}, \ldots, Y_{t}^{n}\right)$.

\section{The scaling limit of Fomin's identity}

The SLE measure is expected to be the limit of discrete critical processes. One example that is well understood at this point is the case of the loop-erased random walk (LERW) which is the prototypical model for the boundary scaling exponent $b=1$. We will describe the discrete model in this section and some different ways in which one can take the limit to get the SLE measure with $b=1$ (corresponding to $\kappa=2$ ).

In 4], Fomin proved an identity showing that a certain probability for loop-erased random walk on $\mathbb{Z}^{2}$ can be given in terms of a determinant of hitting probabilities for simple random walk. He also conjectured that this identity holds for continuous processes. In fact, he wrote,

"... we do not need the notion of loop-erased Brownian motion. Instead, we discretize the model, compute the probability, and then pass to the limit."

It is shown in [6] that the scaling limit of the determinant of hitting probabilities for simple random walk is, in fact, the determinant of the hitting densities of Brownian excursions, i.e., the so-called excursion Poisson kernel determinant.

We conclude this section by deriving the scaling limit of Fomin's identity directly in the case $n=2$ using the ideas of the previous sections, and show how the probability that an $\mathrm{SLE}_{2}$ avoids a Brownian excursion may be computed in terms of the excursion Poisson kernel determinant. This is the natural continuous analogue of the discrete $n=2$ case, in which Fomin's identity gives the probability that a loop-erased walk avoids a simple random walk in terms of the determinant of the simple random walk hitting matrix.

\subsection{Loop-erased random walk}

We write the integer lattice $\mathbb{Z}^{2}$ in complex form $\mathbb{Z}+i \mathbb{Z}$. If $A \subsetneq \mathbb{Z}^{2}$, we let $\partial A=\{z \in$ $\left.\mathbb{Z}^{2}: \operatorname{dist}(z, A)=1\right\}$. We call $\omega=\left[\omega_{0}, \ldots, \omega_{n}\right]$ a (nearest neighbor) random walk path if $\omega_{0}, \ldots, \omega_{n} \in \mathbb{Z}^{2}$ with $\left|\omega_{j}-\omega_{j-1}\right|=1$ for $j=1, \ldots, n$. We write $|\omega|=n$ for the number of steps in the path. If $z, w \in \partial A$, we say that $\omega$ is an excursion in $A$ from $z$ to $w$ if $\omega_{0}=z, \omega_{n}=w$, and $\omega_{1}, \ldots, \omega_{n-1} \in A$. The (simple random walk) excursion measure $\nu(A ; z, w)$ is the measure that assigns weight $4^{-|\omega|}$ to each excursion $\omega$ from $z$ to $w$ in $A$. We write $h_{A}(z, w)=|\nu(A ; z, w)|$. The loop-erased excursion measure $\nu_{L E}(A ; z, w)$ is the measure obtained from $\nu(A ; z, w)$ by doing chronological loop-erasure on each excursion. Note that $\left|\nu_{L E}(A ; z, w)\right|=|\nu(A ; z, w)|=h_{A}(z, w)$. We also write $\nu^{\#}(A ; z, w)=\nu(A ; z, w) / h_{A}(z, w)$, and similarly for $\nu_{L E}^{\#}(A ; z, w)$, to denote the measures normalized to be probability measures. 
Let $\mathcal{H}=\left\{j+i k \in \mathbb{Z}^{2}: k>0\right\}$ denote the discrete upper half plane with $\partial \mathcal{H}=\mathbb{Z}$. It is not difficult to see (cf., equation $(28)$ in [6] ) that if $j_{1} \neq j_{2}$, then

$$
h_{\mathcal{H}}\left(j_{1}, j_{2}\right)=\frac{1}{16} G_{\mathcal{H}}\left(j_{1}+i, j_{2}+i\right)
$$

where $G_{\mathcal{H}}$ denotes the random walk Green's function in $\mathcal{H}$. Indeed we get the expression above by dividing each excursion from $j_{1}$ to $j_{2}$ in $\mathcal{H}$ into its first step $\left(j_{1}, j_{1}+i\right)$; its middle, i.e., any walk in $A$ starting at $j_{1}+i$ and ending at $j_{2}+i$; and its final step $\left(j_{2}+i, j_{2}\right)$. It is not hard to show (cf., equation (15.3) in [17]) that

$$
G_{\mathcal{H}}(x, y)=a(x, \bar{y})-a(x, y)
$$

where $a(x, y)=a(y-x)$ denotes the potential kernel for random walk and $\bar{y}$ denotes the complex conjugate of $y$. The potential kernel has asymptotic behavior

$$
a(z)=\frac{2}{\pi} \log |z|+C+O\left(|z|^{-2}\right)
$$

for a known constant $C$. Also, this relation can be differentiated, i.e.,

$$
a(z-2 i)-a(z)=\frac{2}{\pi}[\log |z-2 i|-\log |z|]+O\left(|z|^{-3}\right) .
$$

Using this, we get the relation

$$
h_{\mathcal{H}}\left(j_{1}, j_{2}\right)=\frac{1}{4 \pi\left(j_{2}-j_{1}\right)^{2}}+O\left(\left(j_{2}-j_{1}\right)^{-3}\right)
$$

Let us consider the scaling limit of this measure. Suppose $N$ is a large integer. Let $\mathcal{H}_{N}=N^{-1} \mathcal{H}$ and let $\nu^{(N)}(j / N, k / N)$ be the measure that assigns measure $4 \pi N^{2} 4^{-|\omega|}$ to each excursion $\omega$ in $\mathcal{H}$ from $j$ to $k$. As $N \rightarrow \infty$, this measure approaches the Brownian excursion measure $\nu_{B}$ on $\mathbb{H}$ defined roughly as follows. The measure $\nu_{B}(x, y)$ has total mass $H_{1}(x, y)=(y-x)^{-2}$. If $\nu_{B}^{\#}(x, y)$ denotes the probability measure obtained by normalization, then this is the measure corresponding to "Brownian motion starting at $x$ conditioned to leave $\mathbb{H}$ at $y$ " which can be made precise using $h$-processes. (See, for example, [5, Section 3.4].)

In the limit above, we fixed an $x, y$ and took the limit. A similar limit can be taken by considering the measure that gives weight $4 \pi 4^{-|\omega|}$ to every excursion (with any possible endpoints). A similar argument shows that the limiting measure obtained is (a constant multiple of) Brownian excursion measure in $\mathbb{H}$ and can be written as

$$
\int_{-\infty}^{\infty} \int_{-\infty}^{\infty} \frac{1}{(y-x)^{2}} \nu_{B}^{\#}(x, y) d x d y
$$

The advantage of this formulation is that it can be used to describe the scaling limit of excursions in Jordan domains without any smoothness assumptions on the boundary; see [5] and [6].

We can also take the scaling limit of the loop-erased measure

$$
\nu_{L E}(\mathcal{H} ; j, k)=h_{\mathcal{H}}(j, k) \nu_{L E}^{\#}(\mathcal{H} ; j, k)
$$


The scaling limit of the normalized measure $\nu_{L E}^{\#}(\mathcal{H} ; j, k)$ was shown in [1] to be $\mathrm{SLE}_{2}$ from $x$ to $y$. (To be honest, the paper [1] considered a radial version, but the basic argument works in this case.)

A rooted (random walk) loop in $A$ is a random walk path $\omega=\left[\omega_{0}, \ldots, \omega_{2 n}\right]$ with $\omega_{0}=\omega_{2 n}$ and $\omega_{1}, \ldots, \omega_{2 n} \in A$. An unrooted (random walk) loop is an equivalence class of loops under the equivalence

$$
\left[\omega_{0}, \omega_{1}, \omega_{2}, \ldots, \omega_{2 n}\right] \sim\left[\omega_{1}, \omega_{2}, \ldots, \omega_{2 n}, \omega_{1}\right] .
$$

For each unrooted loop $\omega$, let $R_{\omega}$ denote the number of distinct rooted loops that produce this unrooted loop. Note that $R_{\omega}$ is always a divisor of $|\omega|$ and the proportion of loops of length $2 n$ with $R_{\omega}<2 n$ decays exponentially in $n$. The random walk loop measure in $A$ is the measure that assigns weight $\left[R_{\omega} /|\omega|\right] 4^{-|\omega|}$ to each unrooted loop in $A$. If we scale the loops by $N^{-1}$ and take the limit, we get the Brownian loop measure [13] as mentioned in Section 2.2

The random walk loop soup in $\mathcal{H}$ is a Poisson point process $\mathcal{C}_{t}$ from the loop measure. At any fixed time $t$, the set $\mathcal{C}_{t}$ is a multi-set of loops, where the number of times the unrooted loop $\omega$ appears has a Poisson distribution with parameter $\left(R_{\omega} /|\omega|\right) 4^{-|\omega|} t$. If $A \subset \mathbb{Z}^{2}$, then $\mathcal{C}_{t}(A)$ denotes the loop soup in $A$, i.e., $\mathcal{C}_{t}$ restricted to loops in $A$. Given a self-avoiding path $\eta=\left[\eta_{0}, \eta_{1}, \ldots, \eta_{k}\right]$ and a realization of the loop soup $\mathcal{C}_{t}$, there is a systematic way of taking the loops in $\mathcal{C}_{1}(A)$ and adding them to $\eta$ to produce a random walk path $\omega$ from $\eta_{0}$ to $\eta_{k}$ whose chronological loop-erasure is $\eta$. It can be described as follows.

- Let $\omega^{1}, \ldots, \omega^{r}$ denote the loops in $\mathcal{C}_{1}(A)$ that intersect $\eta$. It is not difficult to check that (with probability one) there are only a finite number of such loops (assuming $A$ is a proper subset). Order the loops in the order that they appeared in the Poisson process.

- For each $\omega^{j}$ choose a rooted loop that represents it by letting $\eta_{l}$ be the point with smallest index $l$ such that $\eta_{l}$ appears in $\omega^{j}$ and choosing the root of $\omega$ so that the rooted path starts at $\eta_{l}$. If $\omega$ visits $\eta_{l}$ more than once, choose the root uniformly over all the possibilities.

- Attach a loop at $\eta_{l}$ by adding all the loops rooted at $\eta_{l}$ in the order they appear in the Poisson process.

Then straightforward combinatorial arguments show that if $\eta$ is chosen according to the probability measure $\nu_{L E}^{\#}(A ; z, w)$ and $\mathcal{C}_{1}$ is an independent realization of the loop soup in $A$ and the loops are added as above, then the distribution of the resulting random walk is $\nu^{\#}(A ; z, w)$.

Similarly, we can start with $\nu^{\#}(A ; z, w)$ and get independent copies of the loop-erased measure $\nu_{L E}^{\#}(A ; z, w)$ and the loop soup in $A$ restricted to curves that hit the loop-erased path. We will explain this more now. Suppose $\eta=\left[\eta_{0}, \eta_{1}, \ldots, \eta_{k}\right]$ is a self-avoiding excursion in $A$ from $z$ to $w$. The $\nu_{L E}^{\#}(A ; z, w)$ measure of $\eta$ is exactly the $\nu^{\#}(A ; z, w)$ measure of all loops $\omega$ whose loop-erasure is $\eta$. It is straightforward to show (see, for example, Equation (12.2.2) of [7]) that

$$
\nu_{L E}^{\#}(A ; z, w)(\eta)=4^{-|\eta|} \Theta_{A}(\eta) \quad \text { where } \quad \Theta_{A}(\eta)^{-1}=q_{A_{1}}\left(\eta_{1}\right) \cdots q_{A_{k-1}}\left(\eta_{k-1}\right) .
$$


Here $A_{j}=A \backslash\left\{\eta_{1}, \ldots, \eta_{j-1}\right\}$ and $q_{A}(x)$ denotes the probability that a random walk starting at $x$ leaves $A$ before returning to $x$. We will show that

$$
\Theta_{A}(\eta)=\mathbf{P}\left\{\mathcal{C}_{1}(A) \text { contains no loop that intersects } \eta\right\} .
$$

To verify (30), we first note that any path $\omega$ with loop-erasure $\eta$ can be split into a unique fashion as

$$
\omega=\left[\eta_{0}, L_{1}, L_{2}, \ldots, L_{k-1}, \eta_{k}\right]
$$

where $L_{j}$ is a loop rooted at $\eta_{j}$ in $A \backslash\left\{\eta_{1}, \ldots, \eta_{j-1}\right\}$. Each of these loops can be split into a number of ways as

$$
L_{j}=\omega^{j, 1} \oplus \cdots \oplus \omega^{j, r_{j}}
$$

where $\omega^{j, l}$ are loops at $L_{j}$. We give $L_{j}$ weight $4^{-\left|L_{j}\right|}$. We let $\alpha\left(\omega^{j, l}\right)=4^{-\left|\omega^{j, l}\right|} \theta\left(\omega^{j, l}\right)^{-1}$ where $\theta\left(\left[\omega_{0}, \ldots, \omega_{l}\right]\right)$ is the number of $j \geq 1$ such that $\omega_{j}=\omega_{0}$. We give the $l$-tuple $\left(\omega^{j, 1}, \ldots, \omega^{j, l}\right)$ weight

$$
\alpha\left(\omega^{j, 1}\right) \cdots \alpha\left(\omega^{j, l}\right)=\frac{1}{4^{\left|\omega^{j, 1}\right|+\cdots+\left|\omega^{j, r}\right|}\left[\theta\left(\omega^{j, 1}\right) \cdots \theta\left(\omega^{j, l}\right)\right]} .
$$

The following lemma yields (301).

Lemma 5.1. Suppose $A$ is a proper subset of $\mathbb{Z}^{2}$ and $z \in A$. Let $m=m_{z, A}$ denote the measure that assigns weight $4^{-|\omega|} \theta(\omega)^{-1}$ to each loop in $A$ rooted at $z$. Let $\mathcal{A}_{t}$ denote a Poisson point process from this measure and let $l_{t}$ denote the loop obtained by concatenating all the loops in $\mathcal{A}_{t}$ in chronological order. (If there are no loops, then $l_{t}$ is the trivial loop of length 0.) Then

$$
\mathbf{P}\left\{l_{1}=\omega\right\}=4^{-|\omega|} q_{A},
$$

where $q_{A}$ is the probability that simple random walk starting at z leaves $A$ before returning to $z$.

Proof. Let $\mathcal{L}=\mathcal{L}(A, z)$ denote the set of all loops in $A$ rooted at $z$. Note that $1-q_{A}=$ $\sum_{\omega \in \mathcal{L}, \theta(\omega)=1} 4^{-|\omega|}$ so that

$$
\sum_{k=0}^{\infty} \sum_{\omega \in \mathcal{L}, \theta(\omega)=k} 4^{-|\omega|}=\sum_{k=0}^{\infty}\left[\sum_{\omega \in \mathcal{L}, \theta(\omega)=1} 4^{-|\omega|}\right]^{k}=\sum_{k=0}^{\infty}\left(1-q_{A}\right)^{k}=1 / q_{A} .
$$

For the trivial loop $[z], \mathbf{P}\left\{l_{1}=[z]\right\}$ is the probability that there are no loops in the Poissonian realization, i.e.,

$$
\mathbf{P}\left\{l_{1}=[z]\right\}=\exp \left\{-\sum_{\omega \in \mathcal{L}} 4^{-|\omega|} \theta(\omega)^{-1}\right\} .
$$

It now follows from (31) that

$$
\sum_{\omega \in \mathcal{L}} 4^{-|\omega|} \theta(\omega)^{-1}=\sum_{k=1}^{\infty} \frac{1}{k} \sum_{\omega \in \mathcal{L}, \theta(\omega)=k} 4^{-|\omega|}=\sum_{k=1}^{\infty} \frac{1}{k}\left(1-q_{A}\right)^{k}=-\log q_{A}
$$


so therefore

$$
\mathbf{P}\left\{l_{1}=[z]\right\}=q_{A} .
$$

More generally, if $\omega$ is a loop that returns to the origin $k$ times, and $j_{1}, j_{2}, \ldots, j_{r}$ are positive integers summing to $k$, we could get the loop $\omega$ by splitting the loop into $r$ loops where the $m$ th loop is from the $\left(j_{1}+\cdots+j_{m-1}\right)$ th to the $\left(j_{1}+\cdots+j_{m}\right)$ th return to $z$. By considering all these possibilities, one can deduce that

$$
\mathbf{P}\left\{l_{1}=\omega\right\}=q_{A} 4^{-|\omega|} \sum_{j_{1}+\cdots+j_{r}=k} \frac{1}{r !} \frac{1}{j_{1} \cdots j_{r}} .
$$

We leave it to the reader to show that the summation equals one and so $\mathbf{P}\left\{l_{1}=\omega\right\}=q_{A} 4^{-|\omega|}$. (As a hint, write

$$
\frac{1}{1-t}=\exp \{-\log (1-t)\}
$$

and expand both sides in power series in $t$.)

Computing the right side of (30) is very difficult; the local terms corresponding to small loops dominate the probability. However, we can immediately conclude a boundary perturbation rule. If $A \subset A^{\prime}$ and $z, w \in \partial A \cap \partial A^{\prime}$, then

$$
\frac{\Theta_{A^{\prime}}(\eta)}{\Theta_{A}(\eta)}=\mathbf{P}\left\{\mathcal{C}_{1}\left(A^{\prime}\right) \text { contains no loop intersecting both } A^{\prime} \backslash A \text { and } \eta\right\} .
$$

This should be compared to Proposition 3.1.

\subsection{Fomin's identity}

Suppose $A \subset \mathbb{Z}^{2}$ and $x_{1}, \ldots, x_{n}, y_{1}, \ldots, y_{n} \in \partial A$. Fomin 4 showed that the

$$
\nu\left(A ; x_{1}, y_{1}\right) \times \cdots \times \nu\left(A ; x_{n}, y_{n}\right)
$$

measure of the set of $n$-tuples of excursions $\left(\omega^{1}, \ldots, \omega^{n}\right)$ satisfying the condition

$$
L E\left(\omega^{m-1}\right) \cap \omega^{m}=\emptyset, \quad m=2, \ldots, n,
$$

where $L E\left(\omega^{m-1}\right)$ denotes the loop erasure of $\omega^{m-1}$, is given by

$$
\operatorname{det}\left[h_{A}\left(x_{l}, y_{m}\right)\right]_{1 \leq l, m \leq n} .
$$

In the case $A=\mathcal{H}$, topological considerations show that this can be non-zero only if the points appear in order, i.e., after relabeling, they satisfy

$$
x_{1}<\cdots<x_{n}<y_{n}<\cdots<y_{1}
$$

As Fomin points out, since the scaling limit of simple random walk is Brownian motion, which is conformally invariant, this formula in itself suggests the conformal invariance of loop-erased random walk. There are technical difficulties in taking the limit in domains with 
rough boundaries but these can be handled; see [6]. In the case $n=2$, the determinant equals

$$
h_{\mathcal{H}}\left(x_{1}, y_{1}\right) h_{\mathcal{H}}\left(x_{2}, y_{2}\right)\left[1-\frac{h_{\mathcal{H}}\left(x_{1}, y_{2}\right) h_{\mathcal{H}}\left(x_{2}, y_{1}\right)}{h_{\mathcal{H}}\left(x_{1}, y_{1}\right) h_{\mathcal{H}}\left(x_{2}, y_{2}\right)}\right] .
$$

As the differences go to infinity, this is asymptotic to

$$
h_{\mathcal{H}}\left(x_{1}, y_{1}\right) h_{\mathcal{H}}\left(x_{2}, y_{2}\right)\left[1-\frac{\left(y_{1}-x_{1}\right)^{2}\left(y_{2}-x_{2}\right)^{2}}{\left(y_{1}-x_{2}\right)^{2}\left(y_{2}-x_{1}\right)^{2}}\right] \text {. }
$$

If $x_{1}=0, x_{2}=x, y_{2}=y$, then as $y_{1} \rightarrow \infty$ the last expression is asymptotic to $h_{\mathcal{H}}\left(0, y_{1}\right)$ times

$$
h_{\mathcal{H}}(x, y) \frac{x}{y}\left[2-\frac{x}{y}\right]
$$

and we have recovered the expression for $H_{1,1}^{*}(x, y)$ that was derived in Section 3.3 .

\subsection{Review of excursion Poisson kernel determinant}

We now briefly review the necessary notation from [6] about the excursion Poisson kernel. Suppose that $D \subset \mathbb{C}$ is a simply connected Jordan domain and that $\partial D$ is locally analytic at $x$ and $y$. The excursion Poisson kernel is defined as

$$
H_{\partial D}(x, y)=\lim _{\epsilon \rightarrow 0} \frac{1}{\epsilon} H_{D}\left(x+\epsilon \mathbf{n}_{x}, y\right)
$$

where $H_{D}(z, y)$ for $z \in D$ is the usual Poisson kernel, and $\mathbf{n}_{x}$ is the unit normal at $x$ pointing into $D$. Explicit formulæ are known when $D=\mathbb{D}$, the unit disk, or $D=\mathbb{H}$, namely

$$
H_{\partial \mathbb{D}}(x, y)=\frac{1}{\pi|y-x|^{2}}=\frac{1}{2 \pi(1-\cos (\arg y-\arg x))} \quad \text { and } \quad H_{\partial \mathbb{H}}(x, y)=\frac{1}{\pi(y-x)^{2}}
$$

Notice that $\pi H_{\partial \mathbb{H}}(x, y)=H_{\mathbb{H}, 1,1}(x, y)$. Since the excursion Poisson kernel is known [6. Proposition 2.11] to be conformally covariant, we obtain (2) with $b=1, n=1$. Suppose now that $D^{\prime} \subset \mathbb{C}$ is also a Jordan domain and that $f: D \rightarrow D^{\prime}$ is a conformal transformation. Let $x_{1}, \ldots, x_{n}, y_{1}, \ldots, y_{n}$ be distinct boundary points at which $\partial D$ is locally analytic, and assume that $\partial D^{\prime}$ is locally analytic at $f\left(x_{1}\right), \ldots, f\left(x_{n}\right), f\left(y_{1}\right), \ldots, f\left(y_{n}\right)$. It follows 6 , Proposition 2.16] that if $\mathbf{H}_{\partial D}(\mathbf{x}, \mathbf{y})=\left[H_{\partial D}\left(x_{j}, y_{k}\right)\right]_{1 \leq j, k \leq n}$ denotes the $n \times n$ hitting matrix

$$
\mathbf{H}_{\partial D}(\mathbf{x}, \mathbf{y})=\left[\begin{array}{ccc}
H_{\partial D}\left(x_{1}, y_{1}\right) & \cdots & H_{\partial D}\left(x_{1}, y_{n}\right) \\
\vdots & \ddots & \vdots \\
H_{\partial D}\left(x_{n}, y_{1}\right) & \cdots & H_{\partial D}\left(x_{n}, y_{n}\right)
\end{array}\right]
$$

then

$$
\operatorname{det} \mathbf{H}_{\partial D}(\mathbf{x}, \mathbf{y})=\left(\prod_{\ell=1}^{n}\left|f^{\prime}\left(x_{\ell}\right)\right|\left|f^{\prime}\left(y_{\ell}\right)\right|\right) \operatorname{det}\left[H_{\partial D^{\prime}}\left(f\left(x_{j}\right), f\left(y_{k}\right)\right)\right]_{1 \leq j, k \leq n}
$$




\subsection{Direct derivation of the scaling limit}

Suppose that $\gamma$ is an $\mathrm{SLE}_{2}$ in $\mathbb{H}$ from 0 to $\infty$ as in Section 2.1. and for every $0<t<\infty$ let $\mathbb{H}_{t}$ denote the slit-plane $\mathbb{H}_{t}=\mathbb{H} \backslash \gamma[0, t]$. Fix two real numbers $0<x<y<\infty$, and let $\beta:[0,1] \rightarrow \overline{\mathbb{H}}$ be a Brownian excursion from $x$ to $y$ in $\mathbb{H}$. It then follows that

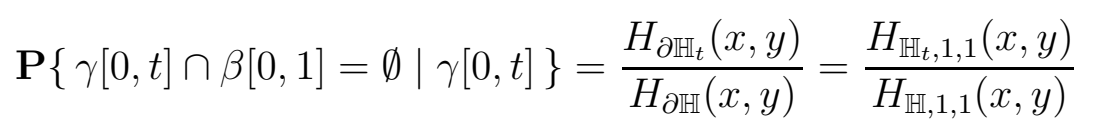

so that taking expectations and limits of (32) gives

$$
\mathbf{P}\{\gamma[0, \infty) \cap \beta[0,1]=\emptyset\}=\tilde{H}_{\mathbb{H}, 1,1}^{*}(x, y)=\frac{x}{y}\left(2-\frac{x}{y}\right) .
$$

The first equality is similar to (14), and the second equality follows from Proposition 3.4.

Theorem 5.2. If $x, y \in \mathbb{R}$ with $0<x<y<\infty$, and that $\gamma, \beta$ are as above, then

$$
\mathbf{P}\{\gamma[0, \infty) \cap \beta[0,1]=\emptyset\}=\frac{\operatorname{det} \mathbf{H}_{\partial \mathbb{D}}(f(\mathbf{x}), f(\mathbf{y}))}{H_{\partial \mathbb{D}}(f(0), f(\infty)) H_{\partial \mathbb{D}}(f(x), f(y))}
$$

where $f: \mathbb{H} \rightarrow \mathbb{D}$ is a conformal transformation.

Remark. By working in $\mathbb{H}$ and $\mathbb{D}$, it is possible to perform explicit calculations. Since the quantity on the right side of (34) is known to be a conformal invariant, we can show, with a combination of conformal transformations, that the probability that an $\mathrm{SLE}_{2}$ avoids a Brownian excursion in $D$ is given by the appropriate determinant of the matrix of excursion Poisson kernels.

Proof. By the scaling rule (16), it suffices without loss of generality to assume that $0<x<1$ and $y=1$. Furthermore, we may assume that the conformal transformation $f: \mathbb{H} \rightarrow \mathbb{D}$ is given by

$$
f(z)=\frac{i z+1}{z+i}
$$

so that $f(0)=-i, f(1)=1, f(\infty)=i$, and

$$
f(x)=\left(\frac{2 x}{x^{2}+1}\right)+i\left(\frac{x^{2}-1}{x^{2}+1}\right)=\exp \left\{-i \arctan \left(\frac{1-x^{2}}{2 x}\right)\right\} .
$$

Notice that $(2 x)^{2}+\left(x^{2}-1\right)^{2}=\left(x^{2}+1\right)^{2}$ so that $|f(x)|=1$ as expected. Writing $f(x)=e^{i \theta}$, we find that

$$
\begin{aligned}
& \frac{\operatorname{det} \mathbf{H}_{\partial \mathbb{D}}(f(\mathbf{x}), f(\mathbf{y}))}{H_{\partial \mathbb{D}}(f(0), f(\infty)) H_{\partial \mathbb{D}}(f(x), f(y))}=\frac{\operatorname{det} \mathbf{H}_{\partial \mathbb{D}}(-i, i, f(x), 1)}{H_{\partial \mathbb{D}}(-i, i) H_{\partial \mathbb{D}}(f(x), 1)} \\
& =\frac{H_{\partial \mathbb{D}}(-i, i) H_{\partial \mathbb{D}}\left(e^{i \theta}, 1\right)-H_{\partial \mathbb{D}}(-i, 1) H_{\partial \mathbb{D}}\left(e^{i \theta}, i\right)}{H_{\partial \mathbb{D}}(-i, i) H_{\partial \mathbb{D}}\left(e^{i \theta}, 1\right)} \\
& =\frac{\frac{1}{2 \pi(1-\cos \pi)} \frac{1}{2 \pi(1-\cos \theta)}-\frac{1}{2 \pi\left(1-\cos \left(\frac{\pi}{2}\right)\right)} \frac{1}{2 \pi\left(1-\cos \left(\frac{\pi}{2}+\theta\right)\right)}}{\frac{1}{2 \pi(1-\cos \pi)} \frac{1}{2 \pi(1-\cos \theta)}} \\
& =\frac{2 \cos \theta+\sin \theta-1}{1+\sin \theta} \text {. }
\end{aligned}
$$


Since $\theta=-\arctan \left(\frac{1-x^{2}}{2 x}\right)$ we see that $\cos \theta=\frac{2 x}{x^{2}+1}$ and $\sin \theta=\frac{1-x^{2}}{x^{2}+1}$ which upon substitution gives

$$
\frac{2 \cos \theta+\sin \theta-1}{1+\sin \theta}=\frac{\frac{4 x}{x^{2}+1}+\frac{1-x^{2}}{x^{2}+1}-1}{1+\frac{1-x^{2}}{x^{2}+1}}=\frac{4 x-2 x^{2}}{2}=x(2-x) .
$$

Comparison with (33) now yields the result.

\section{The $\lambda$-SAW}

Here we will define a one-parameter family of measures on self-avoiding walks on the integer lattice $\mathbb{Z}^{2}$. We conjecture that the scaling limit of this measure will give the measure on SLE as described before.

A simple random walk path $\omega$ that does not visit any point more than once is called a self-avoiding walk. If $\omega$ is an excursion in $A$ connecting boundary points $z, w$, we will call $\omega$ a self-avoiding excursion (SAE). Let $r, \lambda \in \mathbb{R}$ be parameters. If $A \subsetneq \mathbb{Z}^{2}$ and $z, w \in \partial A$, then the measure $W_{r, \lambda, z, w, A}$ is defined to be the measure that assigns to each SAE $\omega$ in $A$ from $z$ to $w$ measure

$$
\exp \left\{-r|\omega|+\lambda m^{*}(A ; \omega)\right\}
$$

where $m^{*}(A ; \omega)$ denotes the random walk loop measure of the set of loops in $A$ that intersect $\omega$. In analogy with the discussion on SLE, we conjecture that for each $\lambda \geq-1 / 2$, there is a critical $r_{\lambda}$ such that the total mass $\left|W_{r_{\lambda}, \lambda, 0, N, \mathcal{H}}\right|$ neither decays nor grows exponentially as $N \rightarrow \infty$.

- If $\lambda=1$ and $e^{-r}=1 / 4$, then $W_{r, 1,0, N, \mathcal{H}}$ is exactly the same as the loop-erased excursion measure $\nu_{L E}(\mathcal{H} ; 0, N)$ which from (29) has total mass asymptotic to $(4 \pi)^{-1} N^{-2}$. Therefore, $e^{-r_{1}}=1 / 4$.

- If $\lambda=0$, then this measure is the same as the usual self-avoiding walk measure that gives each walk of $n$ steps the same measure. In this case $e^{r_{0}}$ is the connective constant for self-avoiding walk which can be defined by saying that the number of SAWs of length $n$ starting at the origin in $\mathbb{Z}^{2}$ grows like $e^{r_{0} n}$.

We now make this conjecture stronger and say that there exist $C>0, b \in \mathbb{R}$ (depending on $\lambda$ ) such that $\left|W_{r_{\lambda}, \lambda, 0, N, \mathcal{H}}\right| \sim C N^{-2 b}$. Consider the measure $N^{2 b-2} C^{-1} W_{r_{\lambda}, \lambda, \cdot, \cdot, \mathcal{H}}$ where we use $\cdot, \cdot$ to mean that we take the union over all $x, y \in \mathbb{R}$. We also scale the paths by $N^{-1}$. Then we conjecture that we have a measure on excursions in $\mathbb{H}$. If $x_{1}<x_{2}<x_{3}<x_{4}$, then the total mass of the set of excursions connecting $\left[x_{1}, x_{2}\right]$ to $\left[x_{3}, x_{4}\right]$ should be

$$
\int_{x_{1}}^{x_{2}} \int_{x_{3}}^{x_{4}} \frac{1}{(t-s)^{2 b}} d t d s
$$

We conjecture that we can write this measure as

$$
\int_{x_{1}}^{x_{2}} \int_{x_{3}}^{x_{4}} \frac{1}{(t-s)^{2 b}} \mu_{\mathbb{H}}^{\#}(s, t) d t d s
$$


where $\mu_{\mathbb{H}}^{\#}(s, t)$ is a conformally invariant probability measure on paths.

We have not discussed the parametrization of the limiting measure. An even stronger conjecture would say that there is an exponent ${ }^{1} \nu$ defined roughly by saying that the typical number of steps in a walk from 0 to $N$ in the measure above contains $N^{1 / \nu}$ steps. Then we could let

$$
\omega^{(N)}(t)=N^{-t} \omega\left(t N^{1 / \nu}\right) .
$$

However, it is not necessary to include the parametrization to have a nontrivial result.

The measure on $k$-tuples of self-avoiding walks is defined similarly, by giving measure

$$
\exp \left\{-r\left(\left|\omega_{1}\right|+\cdots+\left|\omega_{k}\right|\right)+\lambda m^{*}\left(A ; \omega_{1} \cup \cdots \cup \omega_{k}\right)\right\}
$$

We conjecture the following.

- If $\lambda \geq-1 / 2$, then the $\lambda$-SAW gives a limiting measure on paths that is the $\mathrm{SLE}_{\kappa}$ measure. In this case $\kappa, \lambda, b$ are related as before.

- For $\lambda<-1 / 2$, this does not give a limiting measure on simple paths.

For $\lambda=1$, this has been proved using the loop-erased random walk. For $\lambda=0$, this is equivalent to very difficult conjectures about self-avoiding walks; see [12.

\section{Acknowledgements}

The authors would like to express their gratitude to the Fields Institute for their gracious hospitality during the Percolation, SLE, and Related Topics Workshop of September 2005.

\section{References}

[1] M. Bauer, D. Bernard, and K. Kytölä. Multiple Schramm-Loewner Evolutions and Statistical Mechanics Martingales. J. Stat. Phys., 120:1125-1163, 2005.

[2] J. Cardy. Lectures on Conformal Invariance and Percolation. Preprint, 2001. Available online at arXiv:math-ph/0103018.

[3] J. Dubédat. Euler integrals for commuting SLEs. Preprint, 2005. Available online at arXiv:math.PR/0507276. To appear, J. Stat. Phys.

[4] S. Fomin. Loop-erased walks and total positivity. Trans. Amer. Math. Soc., 353:35633583, 2001.

[5] M. J. Kozdron. On the scaling limit of simple random walk excursion measure in the plane. Alea, 2:125-155, 2006.

\footnotetext{
${ }^{1}$ It is traditional to use $\nu$ for this critical exponent. Although we have also used $\nu$ with parameters to denote certain measures on paths, this should not cause confusion.
} 
[6] M. J. Kozdron and G. F. Lawler. Estimates of random walk exit probabilities and application to loop-erased random walk. Electron. J. Probab., 10:1442-1467, 2005.

[7] G. F. Lawler. Loop-erased random walk, in Perplexing Problems in Probability: Festschrift in Honor of Harry Kesten, M. Bramson and R. Durrett, eds., pages 197-247. Birkhäuser, Boston, MA, 1999.

[8] G. F. Lawler. Conformally Invariant Processes in the Plane, American Mathematical Society, Providence, RI, 2005.

[9] G. F. Lawler. The Laplacian- $b$ random walk and the Schramm-Loewner Evolution. Preprint, 2005. To appear, Illinois J. Math.

[10] G. F. Lawler, O. Schramm, and W. Werner. Conformal restriction: the chordal case. J. Amer. Math. Soc., 16:917-955, 2003.

[11] G. F. Lawler, O. Schramm, and W. Werner. Conformal invariance of planar loop-erased random walks and uniform spanning trees. Ann. Probab., 32:939-995, 2004.

[12] G. F. Lawler, O. Schramm, and W. Werner. On the scaling limit of planar self-avoiding walk. In Fractal Geometry and Applications: A Jubilee of Benoît Mandelbrot: Part 2, M. L. Lapidus and M. van Frankenhuijsen, eds., pages 339-364. American Mathematical Society, Providence, RI, 2004.

[13] G. F. Lawler and J. A. Trujillo Ferreras. Random walk loop soup. Preprint, 2004. Available online at arXiv:math.PR/0409291. To appear, Trans. Amer. Math. Soc.

[14] G. F. Lawler and W. Werner. The Brownian loop soup. Probab. Theory Related Fields, 128:565-588, 2004.

[15] N. N. Lebedev. Special Functions 85 their Applications. Dover, New York, NY, 1972.

[16] O. Schramm. Scaling limits of loop-erased random walks and uniform spanning trees. Israel J. Math., 118:221-288, 2000.

[17] F. Spitzer. Principles of Random Walk. Springer-Verlag, New York, NY, second edition, 2001. 